\title{
MESSENGER observations of Mercury's magnetic field structure
}

\author{
Catherine L. Johnson, ${ }^{1,2}$ Michael E. Purucker, ${ }^{3}$ Haje Korth, ${ }^{4}$ Brian J. Anderson, ${ }^{4}$ \\ Reka M. Winslow, ${ }^{1}$ Manar M. H. Al Asad, ${ }^{1}$ James A. Slavin, ${ }^{5}$ Igor. I. Alexeev, ${ }^{6}$ \\ Roger J. Phillips, ${ }^{7}$ Maria T. Zuber, ${ }^{8}$ and Sean C. Solomon ${ }^{9,10}$ \\ Received 23 July 2012; revised 9 October 2012; accepted 16 October 2012; published 14 December 2012.
}

[1] We present a baseline, time-averaged model for Mercury's magnetosphere, derived from MESSENGER Magnetometer data from 24 March to 12 December 2011, comprising the spacecraft's first three Mercury years in orbit around the innermost planet. The model, constructed under the approximation that the magnetospheric shape can be represented as a paraboloid of revolution, includes two external (magnetopause and magnetotail) current systems and an internal (dipole) field and allows for reconnection. We take advantage of the geometry of the orbital Magnetometer data to estimate all but one of the model parameters, and their ranges, directly from the observations. These parameters are then used as a priori constraints in the paraboloid magnetospheric model, and the sole remaining parameter, the dipole moment, is estimated as $190 \mathrm{nT} R_{\mathrm{M}}^{3}$ from a grid search. We verify that the best fit dipole moment is insensitive to changes in the other parameters within their determined ranges. The model provides an excellent first-order fit to the MESSENGER observations, with a root-mean-square misfit of less than $20 \mathrm{nT}$ globally. The results show that the magnetopause field strength ranges from $10 \%$ to $50 \%$ of the dipole field strength at observation locations on the dayside and at nightside latitudes north of $60^{\circ} \mathrm{N}$. Globally, the residual signatures observed to date are dominated by the results of magnetospheric processes, confirming the dynamic nature of Mercury's magnetosphere.

Citation: Johnson, C. L., et al. (2012), MESSENGER observations of Mercury's magnetic field structure, J. Geophys. Res., 117, E00L14, doi:10.1029/2012JE004217.

\section{Introduction}

[2] Mercury is the only inner solar system planet other than Earth to possess a large-scale magnetic field [Ness et al., 1974, 1975]. Vector magnetic field measurements obtained during the first two flybys of Mercury by the

\footnotetext{
${ }^{1}$ Department of Earth, Ocean and Atmospheric Sciences, University of British Columbia, Vancouver, British Columbia, Canada.

${ }^{2}$ Planetary Science Institute, Tucson, Arizona, USA.

${ }^{3}$ NASA Goddard Space Flight Center, Greenbelt, Maryland, USA.

${ }^{4}$ The Johns Hopkins University Applied Physics Laboratory, Laurel, Maryland, USA.

${ }^{5}$ Department of Atmospheric, Oceanic and Space Sciences, University of Michigan, Ann Arbor, Michigan, USA.

${ }^{6}$ Skobeltsyn Institute of Nuclear Physics, Lomonosov Moscow State University, Moscow, Russia.

${ }^{7}$ Planetary Science Directorate, Southwest Research Institute, Boulder, Colorado, USA.

${ }^{8}$ Department of Earth, Atmospheric and Planetary Sciences, Massachusetts Institute of Technology, Cambridge, Massachusetts, USA.

${ }^{9}$ Department of Terrestrial Magnetism, Carnegie Institution of Washington, Washington, D. C., USA.

${ }^{10}$ Lamont-Doherty Earth Observatory, Columbia University, Palisades, New York, USA.

Corresponding author: C. L. Johnson, Department of Earth, Ocean and Atmospheric Sciences, University of British Columbia, 6339 Stores Rd.,

Vancouver, BC V6T 1Z4, Canada. (cjohnson@eos.ubc.ca)

(C)2012. American Geophysical Union. All Rights Reserved. 0148-0227/12/2012JE004217
}

MErcury Surface, Space ENvironment, GEochemistry and Ranging (MESSENGER) spacecraft, together with previous observations from the first and third Mariner 10 flybys, confirmed the presence of a dipolar internal field of dynamo origin [Anderson et al., 2008, 2010; Purucker et al., 2009; Uno et al., 2009]. Although the field is primarily dipolar, and of the same polarity (termed normal polarity) as Earth's field, Mercury's surface magnetic field strength is only about $1 \%$ that of Earth's [Ness et al., 1974], corresponding to a dipole moment that is smaller by a factor of about 1000 . The underlying mechanism for the weak field is not understood, although dynamo models have been proposed in which the thickness of the liquid outer core [Stanley et al., 2005; Heimpel et al., 2005; Takahashi and Matsushima, 2006], the presence of stably stratified layer(s) [Christensen, 2006; Christensen and Wicht, 2008; Manglik et al., 2010], or the precipitation of solid iron [Vilim et al., 2010] play a role. An alternative dynamo mechanism that produces weak fields invokes feedback between the magnetospheric and core dynamo fields [Grosser et al., 2004; Glassmeier et al., 2007a, 2007b; Heyner et al., 2011]. The combined MESSENGER and Mariner 10 flyby data have resulted in estimates of the dipole moment that vary by up to $40 \%$ [Anderson et al., 2010]. This uncertainty reflects trade-offs in estimating both internal and external fields from the limited observations. In particular, the flyby geometries led to a fundamental ambiguity regarding Mercury's large-scale internal field structure: the observations 
could be represented equally well by the field due to either a centered dipole or a somewhat weaker dipole offset northward along the planetary rotation axis [Connerney and Ness, 1988; Anderson et al., 2010; Alexeev et al., 2010]. Prior to MESSENGER's insertion into orbit around Mercury, it was anticipated that it might not be possible to resolve the ambiguity even with orbital observations [Korth et al., 2004], due to the absence of low-altitude magnetic field measurements over the southern hemisphere. However, observations from the MESSENGER Magnetometer (MAG) from the first 88 days in orbit around Mercury have enabled the direct identification of the magnetic equator, indicating a dipole offset of $\sim 0.2 R_{\mathrm{M}}$ northward along the rotation axis [Anderson et al., 2011, 2012], where $R_{\mathrm{M}}(=2440 \mathrm{~km})$ is Mercury's mean radius.

[3] The high solar wind pressure of the inner heliosphere, combined with Mercury's weak dipole moment, results in a magnetosphere that is about eight times smaller than Earth's relative to the planetary diameter. Thus satellite magnetic field observations, taken within even a few hundred kilometers of the surface, contain substantial contributions from fields produced by magnetospheric current systems [see Anderson et al., 2010]. Temporal changes in the solar wind density and speed, and in the interplanetary magnetic field (IMF), lead to variations in Mercury's magnetospheric conditions on timescales of seconds to a Mercury year (88 Earth days). The location of the magnetopause and magnetotail currents are governed by the origin and tilt of the planetary dipole field, so understanding Mercury's magnetic environment requires knowledge of both the internal and external large-scale fields.

[4] During its first Earth year of orbital operations, the MESSENGER spacecraft was in a highly eccentric orbit with a period of $12 \mathrm{~h}$, a periapsis of $200-600 \mathrm{~km}$ altitude at $\sim 60-70^{\circ} \mathrm{N}$, and an apoapsis altitude of $\sim 15,000 \mathrm{~km}$ [see Zurbuchen et al., 2011, Figure 1]. These altitude variations present challenges to analyzing the internal field but have the advantage that every orbit transits into, and out of, the magnetosphere. This geometry allows identification of the bow shock and magnetopause on every orbit, as well as measurement of the IMF conditions before, and after, each magnetospheric transit. After one Mercury year, MAG measurements sampled the northern magnetosphere at all local times and sampled the nightside southern lobe of the magnetotail to distances of almost $5 R_{\mathrm{M}}$. The 88 day annual timescale and associated Mercury solar orbital (MSO) coordinate system govern the geometry of the large-scale external current systems at Mercury, such as the magnetopause and magnetotail currents. The origin of the MSO coordinate system is at the planetary center of mass, $+X$ is sunward, $+Y$ lies in the orbital plane, perpendicular to $+X$ and opposite to the direction of planetary orbital motion (i.e., $+Y$ is positive toward dusk), and $+Z$ is normal to the orbital plane and positive northward. With the exception of induction, the Mercury body-fixed (MBF) coordinate system governs the geometry of the internally generated fields at Mercury. Because of Mercury's small obliquity [Margot et al., 2007, 2012], the MBF and MSO $+Z$ axes, and hence MSO and MBF latitudes, are nearly identical. Northern hemisphere coverage at all body-fixed longitudes is achieved once every 59 Earth days (Mercury's sidereal spin period), at spacecraft altitudes from $200 \mathrm{~km}$ to over $1000 \mathrm{~km}$.
Thus, MAG observations taken in orbit around Mercury afford the first opportunity to examine the global structure of the planet's external and internal fields.

[5] Previous models of Mercury's internal and external fields used a variety of approaches, including spherical harmonic analyses (see reviews by Connerney and Ness [1988] and Anderson et al. [2010]), an empirical magnetospheric model developed for Earth [Tsyganenko, 1995; Tsyganenko and Sitnov, 2005] and scaled to Mercury [Korth et al., 2004; Uno et al., 2009; Anderson et al., 2008, 2010], and a paraboloid magnetospheric model [Alexeev et al., 2008, 2010]. Although physics-based simulations are desirable and now possible [e.g., Kabin et al., 2000; Trávniček et al., 2007; Benna et al., 2010], they are still not suited to the practical purpose of parameter searches or inversions involving large quantities of data. Spherical harmonic separation of internal and external fields requires that observations be taken in a current-free region, a situation that is not the case for MESSENGER Magnetometer data [Alexeev et al., 2008, 2010; Anderson et al., 2010]. In addition, from an estimation perspective, the orbit geometry results in strong covariance among even the coefficients of lowest spherical harmonic degree and order [see, e.g., Connerney and Ness, 1988; Korth et al., 2004]. This covariance is not only among coefficients for the internal and external fields individually but is also between the internal and external fields. Thus, because the fundamental physical assumption of a current-free region is violated and because the estimation problem results in covariance among the lowest degree and order internal and external fields, we do not pursue the traditional spherical harmonic separation approach here. As we detail below, MESSENGER observations permit direct identification of the geometry of the magnetospheric current systems and hence the fields produced by those currents, circumventing this severe covariance problem. The different average IMF orientation at Mercury than at Earth and the absence of major terrestrial current systems such as the ring current and fieldaligned currents suggest that scaling of Earth-based empirical models [Tsyganenko, 1995; Tsyganenko and Sitnov, 2005] may not be appropriate. Although a scaled version of the Tsyganenko and Sitnov [2005] model was used by us previously [Anderson et al., 2008, 2010; Uno et al., 2009] to try to estimate and remove the external fields from the Mariner 10 and MESSENGER flyby observations, we noted limitations in this approach [see Anderson et al., 2010, section 4.2], in particular in capturing the tail currents. Removing current system modules from Earth magnetospheric models is problematic because the different modules are not independent, in particular the tail and ring current systems [e.g., Tsyganenko and Sitnov, 2005].

[6] Here we use a paraboloid magnetospheric model to represent the internal dipole, magnetotail, and magnetopause fields at Mercury. The paraboloid magnetospheric model (PMM) is based on the assumption, first put forward by Alexeev and Shabansky [1972], that the planetary dipole field is confined within a model magnetopause approximated by a paraboloid of revolution. The model has been further developed for Mercury and has proven successful in modeling the Mariner 10 and MESSENGER flyby data [Alexeev et al., 2008, 2010]. The magnetospheric field is the sum of contributions from the planetary dipole, the magnetopause shielding currents, and the tail current sheet. In 
addition, reconnection with the IMF is included through a penetration field proportional to a reconnection efficiency parameter. The model formulation is particularly well suited to MESSENGER orbital observations, as these data provide not only measurements inside the magnetosphere but direct observations of the magnetopause and tail current sheet geometry, as well as IMF observations before and after each magnetospheric transit. We do not pursue a comparison of the paraboloid model with other modeling approaches such as a scaled Tsyganenko and Sitnov [2005] model or Gauss separation of internal and external fields. The fundamental limitations of these other approaches outlined above and by Anderson et al. [2010] mean that it is not possible to assess how well they describe the physical system except via an overall misfit of the model to the MAG data away from the regions of current sources. With Mariner 10 and MESSENGER flyby data, Alexeev et al. [2010] demonstrated that the paraboloid model does indeed provide the best fit to the observations. However, more importantly, the validity of the paraboloid model can be assessed in a more powerful way, specifically investigating how well it describes the magnetopause, magnetotail, and internal fields individually (section 4).

[7] In this paper we present a baseline, time-averaged model for Mercury's magnetosphere, using MAG data from 24 March to 12 December 2011, comprising the first three Mercury years of the MESSENGER spacecraft's orbital observations at Mercury. This approach is philosophically different from that of Alexeev et al. [2010], who estimated a common internal field but different external fields and reconnection efficiencies for the times of the first two MESSENGER flybys. We do not attempt here to estimate time dependence in the external fields, but solve instead for time-averaged background internal and external fields. This approach allows us to obtain robust estimates of the external fields from observations during orbits that repeatedly cover the full range of local times and body-fixed longitudes. Alexeev et al. [2010] used an iterative, linearized minimization approach to solve simultaneously for all the model parameters. Here we take advantage of the orbital MAG data to estimate all but one of the model parameters directly from the observations, thus reducing the model covariance that would be inherent in a multiparameter, nonlinear inversion. We estimate both the mean value and upper and lower bounds for each parameter. From the magnetopause and tail current sheet crossings we constrain the geometry of these currents. From observations during spacecraft transits through the southern lobe of the magnetotail we estimate the flux in a single tail lobe. Observations of the magnetic equator resolve the axial dipole-quadrupole ambiguity otherwise inherent in the orbital geometry [Korth et al., 2004; Anderson et al., 2011, 2012] and provide tight constraints on the dipole tilt and orientation. The remaining model parameter, the average dipole moment, is estimated via a grid search. The baseline model is thus tied to direct constraints from the magnetic field observations and provides a description of the first-order signal in the data throughout the magnetosphere. Further refinements in current systems, the planetary field, and time variability can then be readily assessed relative to this baseline model.

[8] Central to this study are the orbital MAG observations, which we describe in some detail in section 2. Examples of data collected during orbits at two different local times illustrate the major magnetospheric boundaries and the identification of the magnetic equator. We provide a brief overview of the PMM model in section 3, and we refer the reader to previous publications for model formulation details [Belenkaya et al., 2005; Alexeev et al., 2008, 2010]. A description of how the PMM parameters and their uncertainties are estimated from the MAG observations is given in section 4 , and the resulting global model is given in section 5. We discuss possible contributions to the observed residual signatures, including static contributions due to limitations of the model itself, dynamic magnetospheric contributions on a variety of timescales, and contributions related to internal field processes in section 6 .

\section{Magnetic Field Observations}

[9] Vector field observations from orbit about Mercury have been acquired from MESSENGER's MAG since 23 March 2011. Inside the magnetosphere, sampling is typically at 20 samples per second with the exception of a few periods during which operational constraints required that the instrument be turned off [Anderson et al., 2007]. The time that MESSENGER spends inside the magnetosphere during each orbit is on average $1 \mathrm{~h}$ when the orbit is close to the terminator and up to $2 \mathrm{~h}$ when the orbit is close to the midnight-noon plane. The latitudinal coverage varies with magnetic local time and MBF longitude. Examples of data taken during orbits close to the dawn-dusk and noonmidnight planes are shown in Figures 1 and 2, plotted in the MSO coordinate system. Data from these orbits illustrate how the major magnetospheric boundaries (the bow shock, magnetopause, and tail current sheet) and the magnetic equator may be identified from the MAG data. Our analyses use the reduced data records (RDRs) that contain $1 \mathrm{~s}$ averages, and standard deviations, of the magnetic field components.

[10] For each orbit the inbound and outbound bow shock and magnetopause were identified. Diagnostics of the inbound bow shock are an increase in magnetic field magnitude and/or an increase in wave activity just upstream of the shock. Diagnostics of the inbound magnetopause are a rotation in the field direction from the IMF direction toward the planetary field direction, and an accompanying decrease in magnetic field fluctuations [Moldovan et al., 2011; R. M. Winslow et al., Mercury's magnetopause and bow shock from MESSENGER observations, submitted to Journal of Geophysical Research, 2012]. The reverse sequences are seen during outbound bow shock and magnetopause crossings (Figures 1 and 2). Inner and outer limits for each boundary position were identified. The spacecraft is continuously within the magnetosphere between the inner edge inbound and inner edge outbound magnetopause crossings. Inside the magnetosphere the signature of a dipolar field is evident: field magnitudes increase with decreasing spacecraft altitude and increasing northern latitude. For orbits close to the terminator, the field is dominated by the $B_{Y}$ and $B_{\mathrm{Z}}$ components. On the ascending (inbound, dawnside) node of the orbit for 1 August 2011 (Figure 1), the $B_{\mathrm{Y}}$ component of the field was positive, and on the descending (outbound, duskside) node of the orbit the $B_{\mathrm{Y}}$ component was negative. At high northern latitudes the $B_{\mathrm{Z}}$ component dominated and 

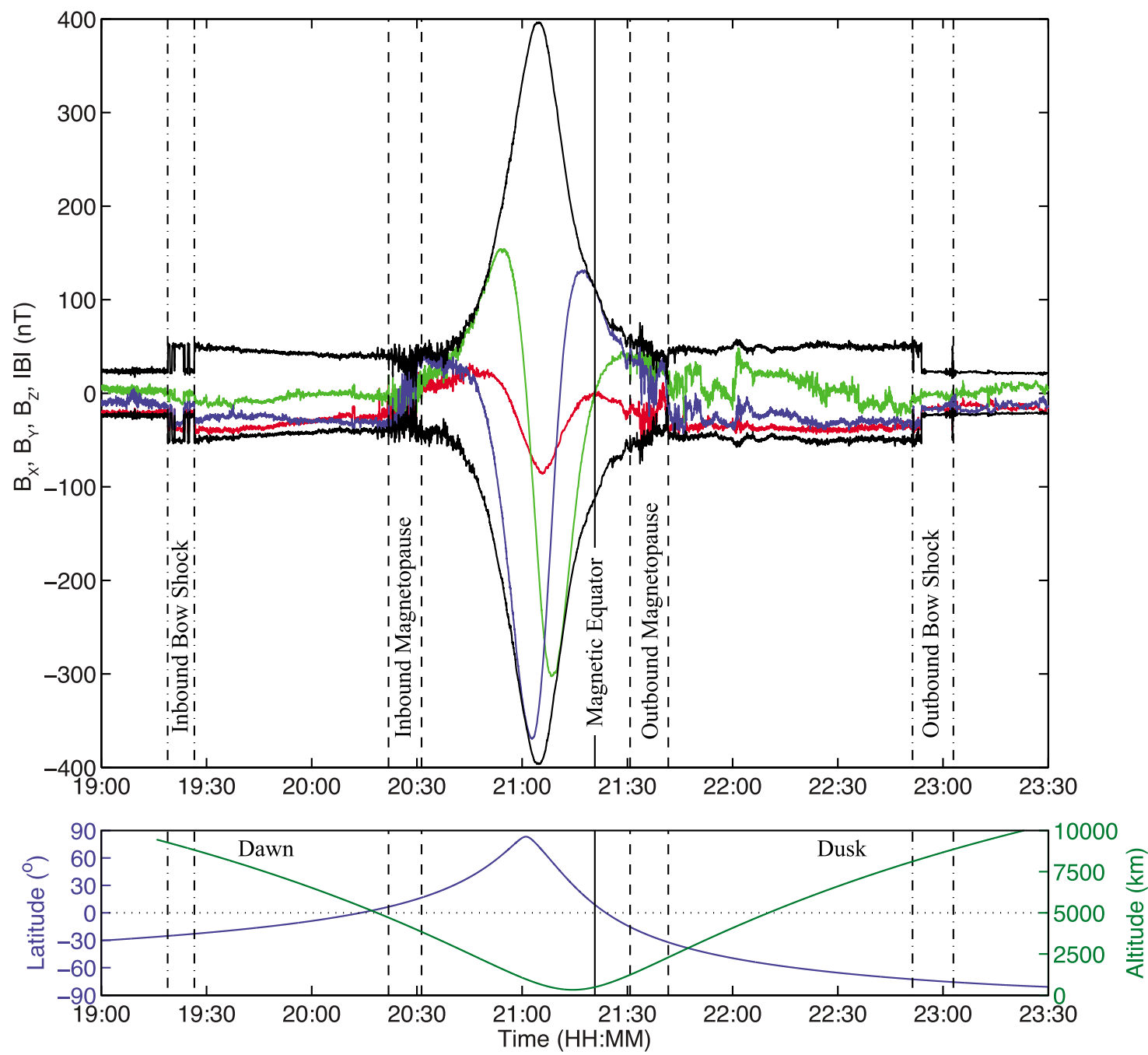

Figure 1. Magnetic field observations in Mercury solar orbital (MSO) coordinates during a dawn-dusk orbit (orbit 274) on 1 August 2011 (UTC). The inbound portion of the orbit was on the dawnside of the planet, close to $06: 00 \mathrm{~h}$ local time and $260^{\circ}$ body-fixed longitude. The outbound portion of the orbit was on the duskside of the planet, close to $18: 00 \mathrm{~h}$ local time and $80^{\circ}$ body-fixed longitude. (top) Magnetic field components $B_{\mathrm{X}}$ (red), $B_{\mathrm{Y}}$ (green), $B_{\mathrm{Z}}$ (blue), and $\pm B$ (black) in $\mathrm{nT}$, where $B$ is the field magnitude. (bottom) The corresponding spacecraft latitude (blue, left ordinate) and altitude (green, right ordinate). The inner and outer magnetopause (dashed lines) and bow shock crossings (dash-dotted lines) are shown. The time of magnetic equator crossing, indicated by the vertical black solid line, occurred northward of the MSO (and planetary geographic) equator (black dotted horizontal line).

was negative. These observations are consistent with the expected field directions in the northern hemisphere for a normal-polarity dipole field.

[11] In contrast, for the midnight-noon orbit on 20 November 2011 (Figure 2), the spacecraft entered the magnetosphere at a downtail distance of $X \sim-4 R_{\mathrm{M}}$. The magnetic field was almost purely antisunward $\left(-B_{\mathrm{X}}\right)$, diagnostic of the southern magnetotail lobe field. Just before 09:30 UTC the spacecraft passed into the northern lobe, as seen by the rotation of the field into the sunward $\left(+B_{\mathrm{X}}\right)$ direction. At about 09:50 UTC, the spacecraft started to measure the planetary dipole field. In this orbit geometry, the dipole field is dominated by the $B_{\mathrm{X}}$ and $B_{\mathrm{Z}}$ components, with $B_{\mathrm{X}}$ positive on the nightside and negative on the dayside, and $B_{Z}$ negative at high northern latitudes. In all, the ascending portion of the orbit accounts for over $80 \%$ of the $2 \mathrm{~h}$ duration of the magnetospheric transit, and the descending dayside portion of the orbit was relatively brief. High-frequency fluctuations were seen throughout the dayside part of this orbit. Large-amplitude fluctuations, accompanied by depressions in magnetic field magnitude at high dayside latitudes, are diagnostic of the northern cusp region [Winslow et al., 2012].

[12] The magnetic equator was identified on an orbit-byorbit basis with a cylindrical coordinate system defined from standard MSO coordinates $\left(\rho_{\mathrm{MSO}}, \phi_{\mathrm{MSO}}, Z_{\mathrm{MSO}}\right)$, where $Z_{\mathrm{MSO}}$ is positive northward, $\rho_{\mathrm{MSO}}=\left(X_{\mathrm{MSO}}^{2}+Y_{\mathrm{MSO}}^{2}\right)^{1 / 2}$ is positive outward and parallel to the projection of the radial direction in the $X_{\mathrm{MSO}}-Y_{\mathrm{MSO}}$ plane, and $\phi_{\mathrm{MSO}}$ is the azimuth angle and is positive eastward and zero at the subsolar point. The magnetic equator on each orbit is the location where the 


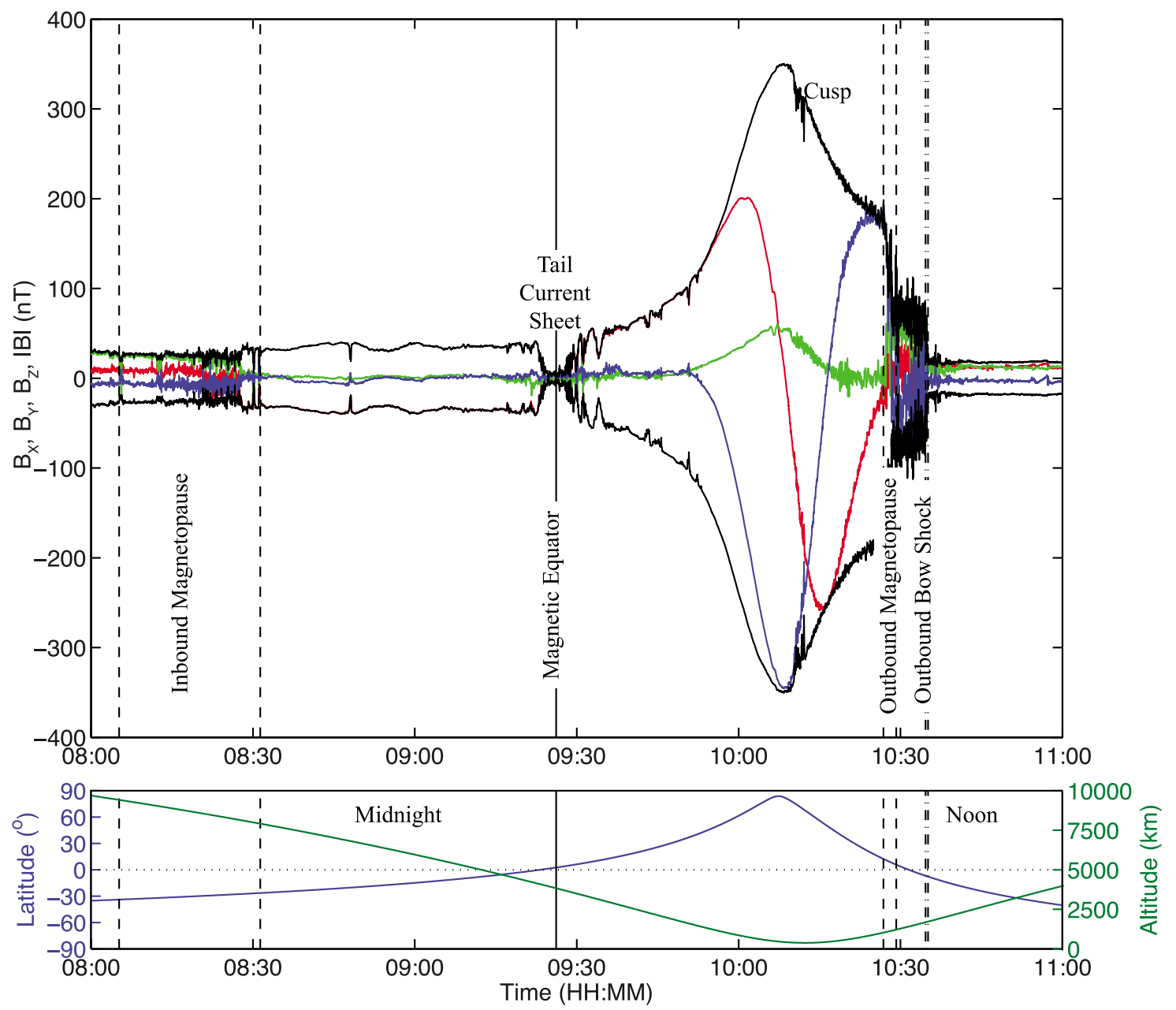

Figure 2. Magnetic field observations in MSO coordinates from a noon-midnight orbit (orbit 497) on 20 November 2011. The inbound portion of the orbit was close to $24: 00 \mathrm{~h}$ local time and $10^{\circ}$ body-fixed longitude. The outbound portion of the orbit was close to 12:00 h local time and $190^{\circ}$ body-fixed longitude. The format follows that of Figure 1 except that the inbound bow shock crossings and inbound magnetosheath are not shown. The spacecraft entered the southern lobe of the magnetotail and spent most of the time inside the magnetosphere in the tail region. The magnetic equator crossing lay in the tail current sheet as seen from the rotation in the magnetic field from $-B_{\mathrm{X}}$ to $+B_{\mathrm{X}}$. The depression in field magnitude and increase in high-frequency variability in the field associated with the rotation in field direction indicates that on this orbit the plasma sheet was of similar width to the current sheet.

component $B_{\rho}$ passes through zero as it changes sign [Anderson et al., 2011, 2012]. The magnetic equator locations for the orbits with data shown in Figures 1 and 2 are indicated. For orbits that enter the magnetosphere in the deep tail region, such as that shown in Figure 2, the magnetic equator lies within the tail current sheet. The current sheet thickness in the $Z$ direction can be estimated from the time interval (and the associated locations) over which the magnetic field rotates from the antisunward southern lobe direction to the sunward northern lobe direction (compare section 4.2).

[13] Observations spanning all body-fixed longitudes and local times are obtained every 59 and 88 days, respectively. The magnetic field strength measured inside the magnetosphere (at latitudes north of $30^{\circ} \mathrm{S}$ ) during the second Mercury year of observations is shown in Figure 3. The signature of the dipole field is evident in the increase in field strength with latitude. Orbit-to-orbit variations in the magnetopause boundary positions are observed. The effects of MESSENGER's highly eccentric orbit are clear: latitudinal coverage depends on local time and spacecraft altitudes range from $200 \mathrm{~km}$ to $10,000 \mathrm{~km}$. Observations below $1000 \mathrm{~km}$ altitude (the approximate subsolar distance to the inner magnetopause crossings) cover almost all northern latitudes for the descending tracks but cover only latitudes north of $\sim 60^{\circ} \mathrm{N}$ for the ascending tracks. Periapsis altitude increases from $\sim 200 \mathrm{~km}$ to $\sim 600 \mathrm{~km}$ during a Mercury year, and the associated decrease in magnetic field strength is clearly seen (Figure 3). For the observations reported here, orbital correction maneuvers were performed every 88 days to lower periapsis altitude to $\sim 200 \mathrm{~km}$. An example of one orbitcorrection maneuver (OCM) is shown in Figure 3. The overall consistency of the observations over one Mercury year, as well as the consistency from one Mercury year to the 

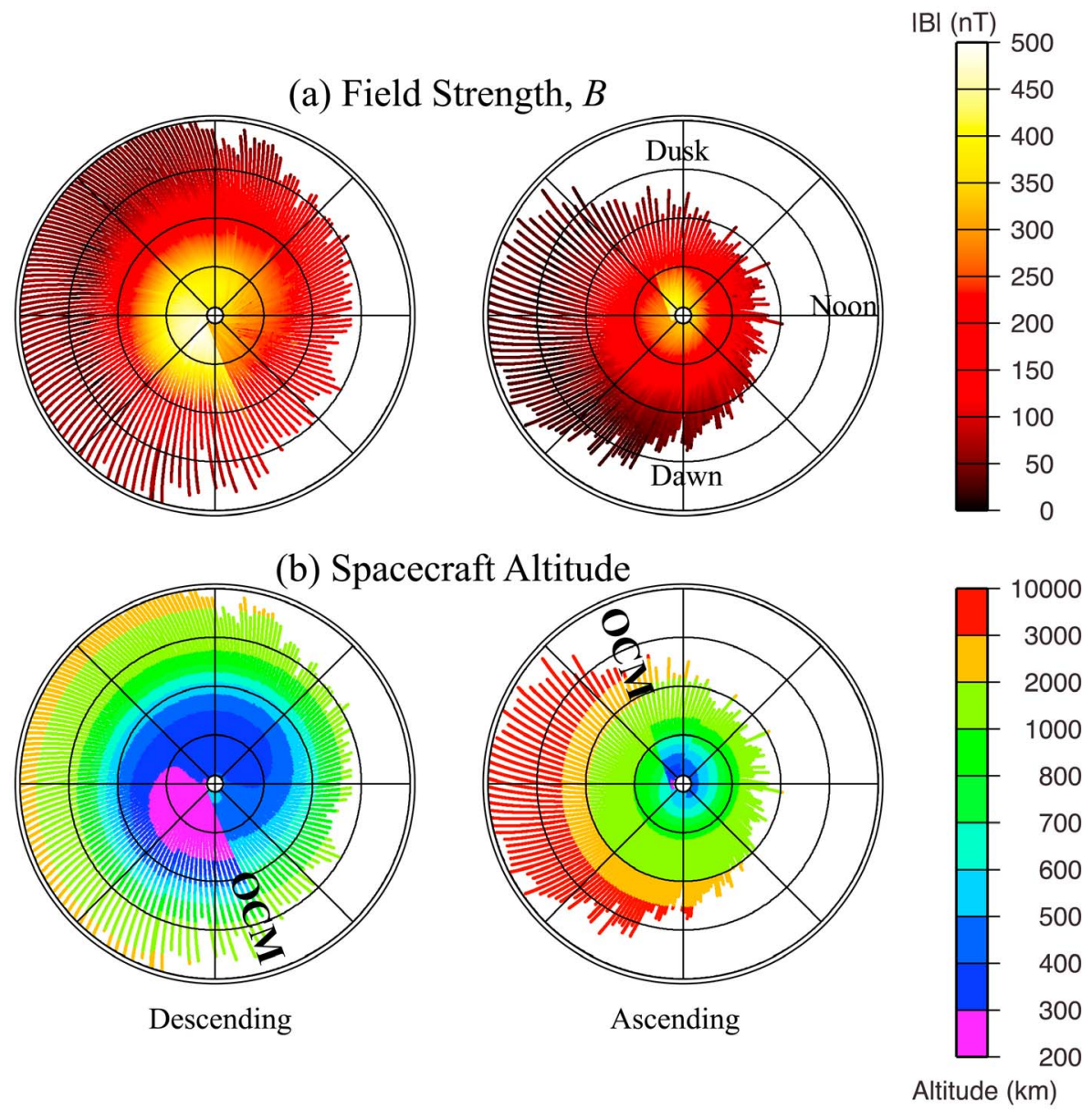

Figure 3. Stereographic projections in aberrated MSO coordinates of (a) magnetic field magnitude, $B$, and (b) spacecraft altitude inside the magnetosphere for (left) descending and (right) ascending tracks from the second Mercury year of observations (20 June to 15 September 2011). Magnetic local noon is to the right. Plots show observations north of $30^{\circ} \mathrm{S}$; grid lines are every $30^{\circ}$ in latitude and every $3 \mathrm{~h}$ in local time. The altitude scale is nonlinear; note the large variations in spacecraft altitude, especially during the ascending portions of each orbit. The increase in periapsis altitude during a Mercury year is evident, and OCM marks an orbit-correction maneuver to lower periapsis altitude to $\sim 200 \mathrm{~km}$.

next, motivates the generation of a baseline average model for Mercury's magnetosphere.

\section{Paraboloid Magnetospheric Model}

[14] The PMM [Alexeev and Shabansky, 1972] specifies a model magnetopause that is a paraboloid of revolution and confines the planetary dipole field and the field from the dawn-to-dusk-flowing magnetotail current. The full model field is the superposition of the dipole, magnetopause, and tail fields. Reconnection with the IMF, $\mathbf{B}_{\mathrm{IMF}}$, can be represented via a reconnection "efficiency" parameter, $k$, where 0 $\leq k \leq 1$, such that $k \mathbf{B}_{\mathrm{IMF}}$ is added to the paraboloid magnetospheric model field. The reconnection field requires no change to the magnetopause current system. The full model formulation, as applied here, has been described by Belenkaya et al. [2005] and Alexeev et al. [2008, 2010].

[15] The currents on the magnetopause and in the magnetotail are organized by the dipole origin and orientation - the Mercury solar magnetic (MSM) coordinate system. In general, the MSM coordinate system is related to the MSO system via a displacement (specified by the dipole origin relative to the planetary origin) and a rotation about the $X_{\text {MSO }}$ axis (governed by the tilt and azimuth) [Alexeev et al., 2010]. The dipole and magnetopause fields are obtained by solving Laplace's equation in parabolic coordinates $(\alpha, \beta$, $\phi)$, under the assumption that the region between the planetary surface and the magnetopause is current free. In the absence of reconnection of the IMF with the planetary field, the component of the magnetic field normal to the magnetopause is zero. The magnetopause is defined as the parabolic surface on which $\beta=1$. In the $X_{\mathrm{MSM}}-Z_{\mathrm{MSM}}$ plane, the tail current sheet is confined within the parabola, $\beta=\beta_{0}$.

[16] The PMM is specified by a total of 12 parameters (Table 1). The basic geometry of the model magnetopause and magnetotail is shown in Figure 4. Variable magnetopause flaring $(\gamma \neq 1)$ is included in the model formulation 
Table 1. Parameters in Magnetospheric Models, Their Average Values, and the Range in Mean Values Derived From MAG Observations

\begin{tabular}{|c|c|c|c|c|c|}
\hline Symbol & Unit & Mean & Range & Description & $\begin{array}{l}\text { Parameter Estimation Procedure } \\
\text { (Corresponding Text Section) }\end{array}$ \\
\hline$R_{\mathrm{SS}}$ & $R_{\mathrm{M}}$ & 1.45 & $1.4-1.5$ & $\begin{array}{l}\text { loid Magnetospheric Model } \\
\text { Subsolar magnetopause } \\
\text { distance }\end{array}$ & $\begin{array}{l}\text { Paraboloid fit to magnetopause crossings } \\
\text { (section 4.2) }\end{array}$ \\
\hline$\gamma$ & None & 1.0 & Upper bound of 1.1 & $\begin{array}{l}\text { Magnetopause paraboloid } \\
\text { flaring parameter }\end{array}$ & Set $=1($ section 4.2$)$ \\
\hline$R_{2}$ & $R_{\mathrm{M}}$ & 1.41 & $1.36-1.46$ & $\begin{array}{l}\text { Distance to inner edge of } \\
\text { cross-tail current sheet } \\
\text { (closest approach to the } \\
\text { planet in } X \text { - } Z \text { plane) }\end{array}$ & $\begin{array}{l}\text { Current sheet was on average tailward of near- } \\
\text { planet equator crossings; } R_{2} \text { from radial } \\
\text { distance to near-planet equator crossings within } \\
\pm 3 \text { h of midnight (section } 4.3 \text { ) }\end{array}$ \\
\hline$D_{\mathrm{D}}$ & $R_{\mathrm{M}}$ & 0.09 & $0.06-0.14$ & $\begin{array}{l}\text { Half thickness of the cross- } \\
\text { tail current sheet }\end{array}$ & $\begin{array}{l}\text { Identify rotation in magnetic field associated } \\
\text { with the tail current sheet in far tail (section 4.3) }\end{array}$ \\
\hline$B_{\mathrm{T}}$ & nT & 137 & $123-152$ & $\begin{array}{l}\text { Model magnetotail field } \\
\text { intensity }\end{array}$ & $\begin{array}{l}\text { Estimated from } F \text { (average flux in a single tail } \\
\left.\text { lobe), } R_{2} \text {, and } D_{\mathrm{D}} \text { (section } 4.3\right)\end{array}$ \\
\hline$X_{0}, Y_{0}, Z_{0}$ & $R_{\mathrm{M}}$ & $0,0,0.196$ & $0,0,0.192-0.200$ & $\begin{array}{l}\text { Center position of } \\
\text { planetary dipole relative to } \\
\text { planetary center along } \\
X_{\mathrm{MSO}}, Y_{\mathrm{MSO}}, Z_{\mathrm{MSO}} \text { axes }\end{array}$ & $\begin{array}{l}\text { Set } X_{0}=Y_{0}=0 \text { on the basis of axisymmetry of } \\
\text { field about rotation axis; } Z_{0} \text { established from } \\
\text { northward offset of magnetic equator (section 4.1) }\end{array}$ \\
\hline$\Theta, \Phi$ & deg & 0,0 & $\begin{array}{l}\text { Tilt upper bound } \\
\text { of } 1^{\circ} \text { at } 283^{\circ} \mathrm{E}\end{array}$ & $\begin{array}{l}\text { Tilt and azimuth of dipole } \\
\text { relative to rotation axis. }\end{array}$ & $\begin{array}{l}\text { Magnetic equator analyses and axisymmetry of } \\
\text { field about rotation axis (section } 4.1 \text { ) }\end{array}$ \\
\hline $\mathrm{m}$ & $\mathrm{nT} R_{\mathrm{M}}^{3}$ & 190 & See text & $\begin{array}{l}\text { Magnitude of planetary } \\
\text { dipole moment }\end{array}$ & $\begin{array}{l}\text { Grid search using paraboloid model to } \\
\text { minimize misfit to observations (sections } 4.5 \\
\text { and 5) }\end{array}$ \\
\hline$k$ & None & 0 & Not considered & $\begin{array}{l}\text { Magnetopause } \\
\text { reconnection efficiency }\end{array}$ & Set $=0($ section 4.4$)$ \\
\hline $\begin{array}{l}R_{\mathrm{SS}} \\
\alpha\end{array}$ & $\begin{array}{l}R_{\mathrm{M}} \\
\text { None }\end{array}$ & $\begin{array}{l}1.45 \\
0.5\end{array}$ & & $\begin{array}{l}\text { ue Magnetopause Model } \\
\text { Subsolar distance } \\
\text { Tail flaring }\end{array}$ & Fit to magnetopause crossings \\
\hline
\end{tabular}

[Belenkaya et al., 2005], such that the magnetopause surface $\left(X_{\mathrm{MP}}, Y_{\mathrm{MP}}, Z_{\mathrm{MP}}\right)$ is given by the paraboloid $\beta=1$, where

$$
\begin{gathered}
\left(\frac{Z_{\mathrm{MP}}}{R_{1}}\right)^{2}+\left(\frac{Y_{\mathrm{MP}}}{R_{1}}\right)^{2}+2\left(\frac{X_{\mathrm{MP}}}{R_{1}}\right)=\left(\gamma^{2}+1\right) \\
R_{1}=\frac{2 R_{\mathrm{SS}}}{\gamma^{2}+1} .
\end{gathered}
$$

$R_{1}$ is a scale length that determines the magnetopause size. For $\gamma=1, R_{1}=R_{\mathrm{SS}}$, the subsolar magnetopause distance, as shown in Figure 4. Values of $\gamma$ greater than 1 correspond to a less flared magnetopause.

[17] The magnetotail is bounded by the surface $\beta_{0}$, given by

$$
\beta_{0}=\frac{D_{\mathrm{D}}}{R_{1}}\left(\frac{2 R_{2}}{R_{1}}+\gamma^{2}\right)^{-\left(\frac{1}{2}\right)}
$$

where $D_{\mathrm{D}}$ and $R_{2}$ are the half thickness and distance (in the $X_{\mathrm{MSM}}-Z_{\mathrm{MSM}}$ plane) to the inner edge of the cross-tail current sheet, respectively, and the flux, $F$, in one lobe of the tail is

$$
F=B_{\mathrm{T}} R_{1}^{2}\left\{\cos ^{-1} \beta_{0}+\frac{1}{3}\left[2-\left(2+\beta_{0}\right) \sqrt{\left(1-\beta_{0}^{2}\right)}\right]\right\} .
$$

Thus, the tail field parameter, $B_{\mathrm{T}}$, depends linearly on the tail flux, $F$, and nonlinearly on the other magnetopause and magnetotail parameters, $R_{\mathrm{ss}}, \gamma, R_{2}$, and $D_{\mathrm{D}}$. We checked that the normal field at the magnetopause boundary $\left(B_{\beta}\right)$ is zero in our numerical implementation of the model, when the reconnection efficiency, $k$, is set to zero. Specifically, we confirmed that the $B_{\beta}$ component of the field was less than $2 \times 10^{-5} \mathrm{nT}$ along the entire magnetopause from the subsolar point to $6 R_{\mathrm{M}}$ downtail. The ratio $B_{\beta} / B$, where $B$ is the total field strength, was less than $0.001 \%$. MSO positions at the magnetopause and inside the magnetosphere were corrected for solar wind aberration [Paschmann and Daly, 1998]. An aberration angle was calculated for each MESSENGER orbit using Mercury's instantaneous orbital speed and a mean solar wind speed of $405 \mathrm{~km} / \mathrm{s}$ from the ENLIL model for the period 24 March to 12 December 2011 [Odstrcil, 2003].

\section{Analyses: Estimation of Model Parameters From Orbital Observations}

[18] As outlined in section 2, we have used orbital MAG data to identify the magnetopause, the tail current sheet, and the magnetic equator. Here we describe how we used these observations to estimate parameters in the PMM (Table 1). Our procedure differs from the approach of Alexeev et al. [2010], who solved simultaneously for a common internal field but different external fields and reconnection efficiencies for the times of the first two MESSENGER flybys. Although that approach provided a good fit to the observations, there was substantial covariance among the model parameters, as can be seen, for example, for the tail and magnetopause fields from equations (1)-(4) and as 


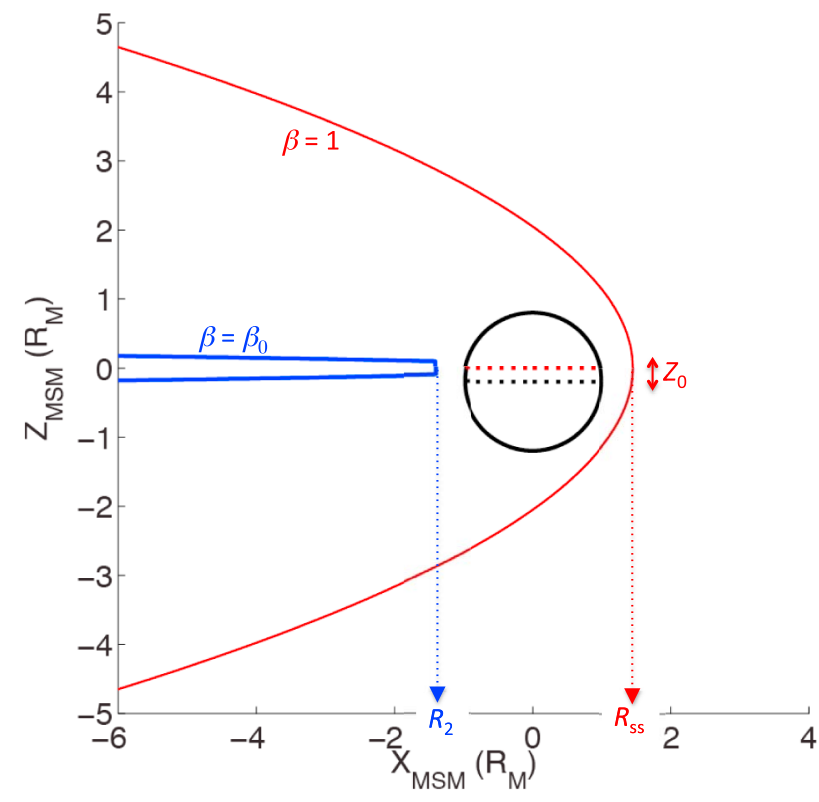

Figure 4. Cross section in the $X_{\mathrm{MSM}}-Z_{\mathrm{MSM}}$ plane of the magnetopause and magnetotail current regions in the paraboloid magnetospheric model. $R_{\mathrm{SS}}$ is the magnetopause subsolar distance, and $R_{2}$ is the distance to the inner edge of the tail current sheet. At $R_{2}$ the current sheet half thickness is $D_{\mathrm{D}}$. The magnetopause is the $\beta=1$ paraboloid of revolution about the $X_{\mathrm{MSM}}$ axis; the parameter $\gamma$ controls the flaring $(\gamma=1)$. The tail current sheet is symmetric about the $Z_{\mathrm{MSM}}=0$ plane. In an $X_{\mathrm{MSM}}-Z_{\mathrm{MSM}}$ plane the boundary of the current sheet tailward of $R_{2}$ is described by the $\beta=\beta_{0}$ parabola. The black circle denotes the planetary outline. At Mercury the dipole origin is offset northward by $Z_{0}$ from the planetary center as shown in the sketch.

discussed by Alexeev et al. [2010]. Data acquired in orbit allowed us to derive the PMM parameters and their uncertainties for the planetary field, magnetopause, and tail current sheet directly from the observations. Importantly, with the exception of the tail flux, which depends on both the tail field and the shape of the magnetopause in the tail region, the parameters derived in this way are independent of each other. These parameters were then used as a priori constraints in the PMM model, and the remaining parameter, the dipole moment, was estimated via a grid search. This approach avoids trade-offs among the model parameters inherent in a minimization approach, provides quantitative measures of the variability in these parameters, and allows us to assess whether the functional forms implicit in the model may need to be adjusted.

\subsection{Dipole Offset and Orientation}

[19] We first summarize observations that constrain the dipole offset and orientation, since these parameters (together with the dipole moment; see section 4.4) control the location of the major magnetospheric current systems. MAG observations from MESSENGER's first 88 days in orbit indicated that Mercury's magnetic equator is offset northward from the planetary center [Anderson et al., 2011]. The results of Anderson et al. [2012] confirm this northward offset of the dipole: the mean offset calculated from the first three Mercury years of data is $479 \pm 9 \mathrm{~km}$ (Table 1 ). There is no evidence for an offset in the $X$ and $Y$ directions, so we set the dipole origin PMM parameters to be $X_{0}=Y_{0}=0$ and $Z_{0}=0.196 R_{\mathrm{M}} \pm 0.004 R_{\mathrm{M}}$ (Table 1). Anderson et al. [2012] reported a mean value for the offset of $477 \pm 7 \mathrm{~km}$ on the basis of data that extend into the fourth Mercury year of observations, but we restrict ourselves to the statistic derived from the first 3 years of data for consistency with the remaining analyses discussed here. The magnetic equator observations constrain the tilt in the field to be a maximum of $0.8^{\circ}$ with an azimuth of the southern hemisphere pole of $283^{\circ} \mathrm{E}$ [Anderson et al., 2012, Figure 5], confirming the apparent axisymmetry of the field seen in Figure 3 and observed in the MESSENGER flyby data [Anderson et al., 2010; Uno et al., 2009]. We thus set the tilt and azimuth of the dipole to be zero with an upper bound of $1^{\circ}$ tilt at $283^{\circ} \mathrm{E}$ (Table 1).

\subsection{Magnetopause Subsolar Distance and Flaring}

[20] Fields due to currents on the magnetopause depend on the internal dipole field and the magnetopause shape. We fit a paraboloid of revolution (Figure 5 and Table 1) to the inbound and outbound magnetopause crossings identified on each magnetosphere pass (Winslow et al., submitted manuscript, 2012), after accounting for the northward offset of the dipole (see Anderson et al. [2011] and section 4.3). To first order the magnetopause is a figure of revolution, as detailed by Winslow et al. (submitted manuscript, 2012). The

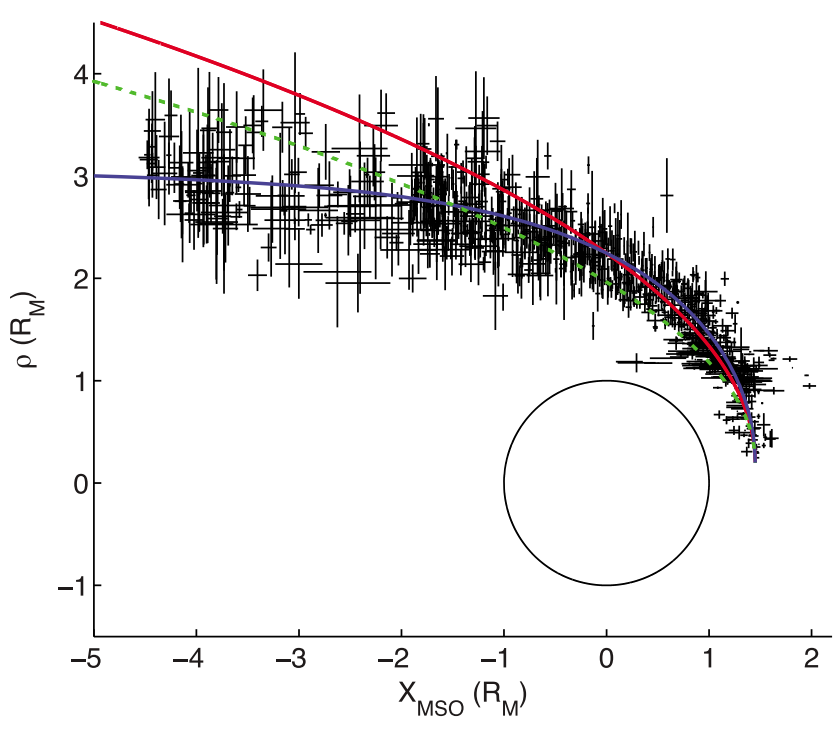

Figure 5. Magnetopause locations, where $\rho=$ $\sqrt{\left(Y_{M S O}^{2}+\left(Z_{M S O}-Z_{0}\right)^{2}\right)}$ and $Z_{0}$ denotes the dipole offset along the rotation axis. Error bars denote the range of distances between the innermost and outermost magnetopause identification on each inbound and outbound crossing (see text). The best fit Shue et al. [1997] model (solid blue line), the nominal paraboloid model (solid red line) used in our magnetospheric model, and the $\gamma=1.3$ paraboloid model (green dashed line) used to explore the effect of magnetopause shape in section 6 are shown. All have subsolar distances $R_{\mathrm{SS}}=1.45 R_{\mathrm{M}}$ (Table 1$)$. 

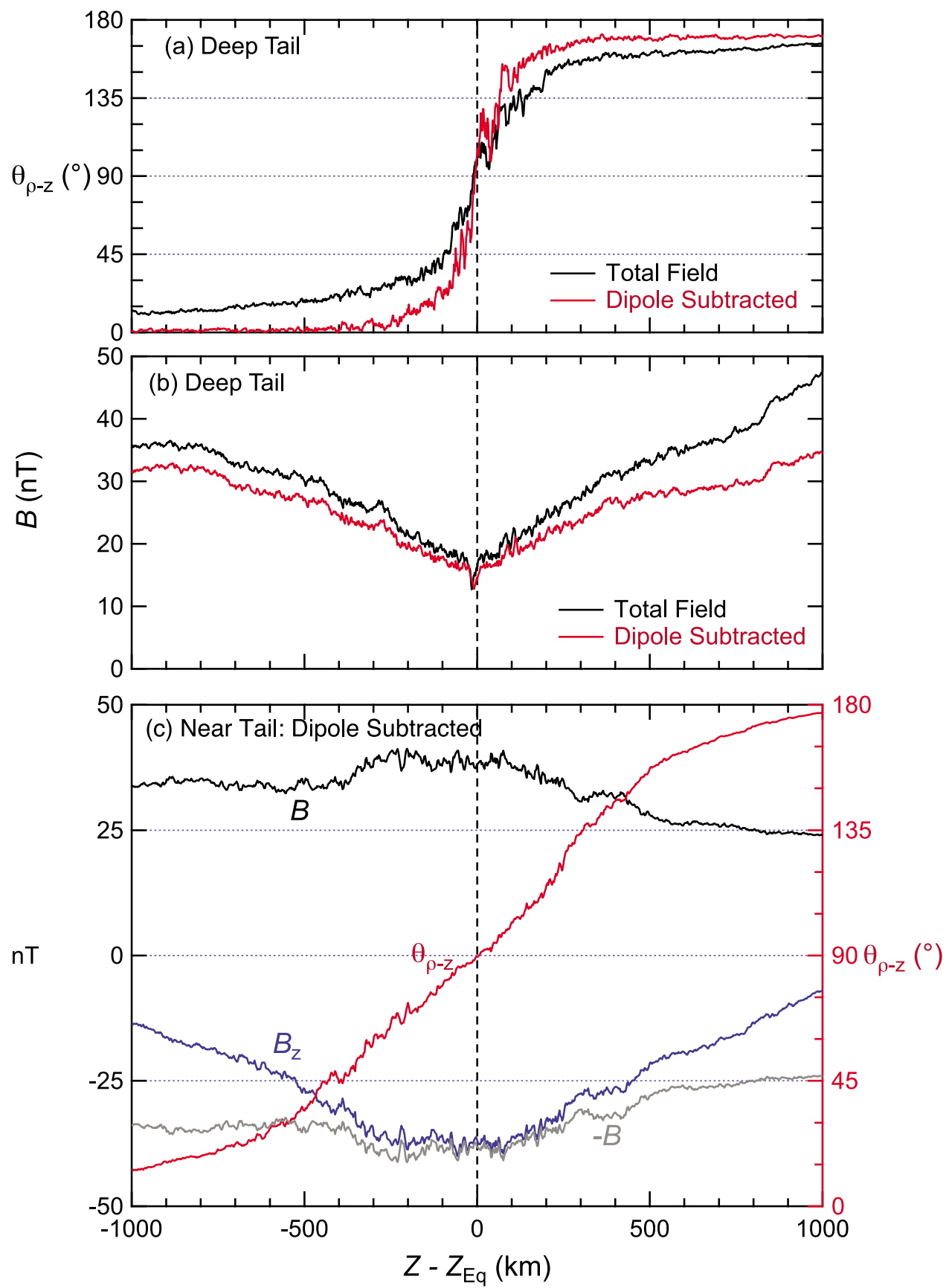

Figure 6. Stacks of measurements from 79 deep-tail current sheet crossings with (red) and without (black) the dipole field removed for (a) tilt, $\theta=\cos ^{-1}\left(B_{\rho} / B_{\rho Z}\right)$ in degrees, where $B_{\rho Z}$ is the magnitude of $\mathbf{B}$ projected into the $\rho-Z$ plane, and (b) field magnitude $(B)$ in nT. Each orbit is aligned on its equator crossing prior to stacking. (c) Stacks for 47 near-tail orbits (see text for selection criteria) showing $\mathbf{B}, B_{\mathrm{Z}}$, and $\theta$ after removal of the dipole field.

crossings are fit well on the dayside and on the nightside close to the planet by a paraboloid with $R_{\mathrm{SS}}=1.45 R_{\mathrm{M}}$ and $\gamma=1$. Although the nightside magnetopause crossings are better fit with a value for $\gamma$ greater than 1, values of $\gamma$ greater than $\sim 1.1$ yield models that provide a poor overall fit to the crossings on the dayside (see, e.g., the curve for $\gamma=1.3$; Figure 5). Thus, we set $\gamma=1$, with an upper bound of 1.1.
For this range of $\gamma$, a range of $R_{\mathrm{SS}}$ values from $1.4 R_{\mathrm{M}}$ to $1.5 R_{\mathrm{M}}$ yield similar root mean square (RMS) misfit values to the near-planet magnetopause crossings, and so we assign an uncertainty of $0.05 R_{\mathrm{M}}$ to the mean $R_{\mathrm{SS}}$ values (Table 1). We also fit the magnetopause crossings with the functional form proposed by Shue et al. [1997] for use in our tail flux calculation (section 4.2); this model provides 


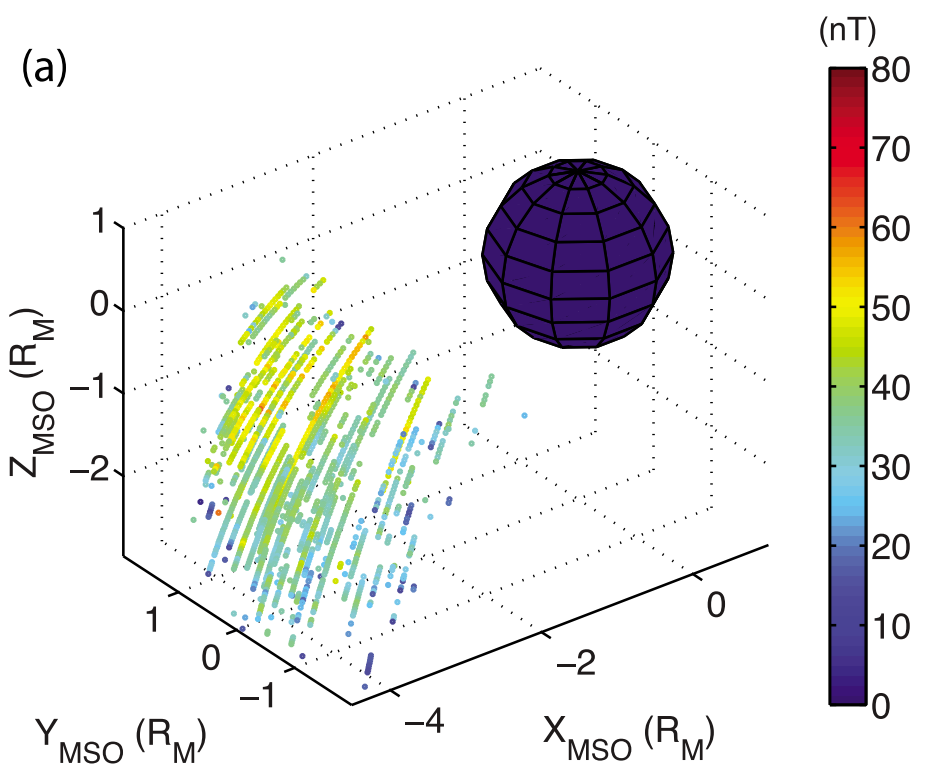

(b)

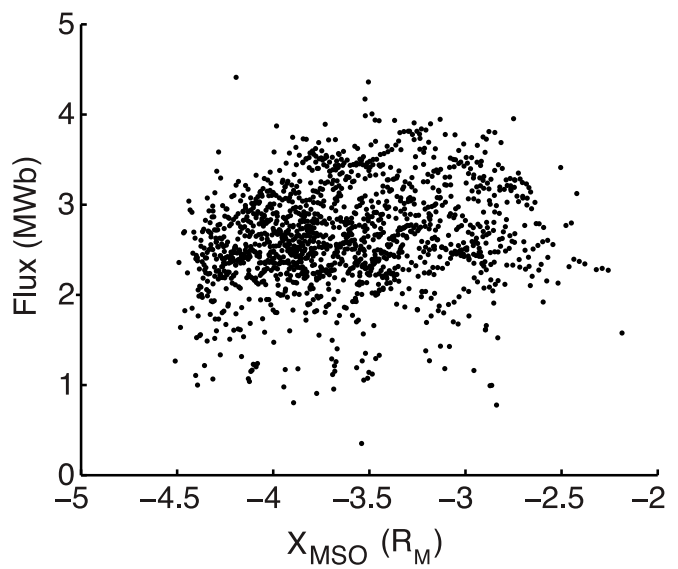

(c)

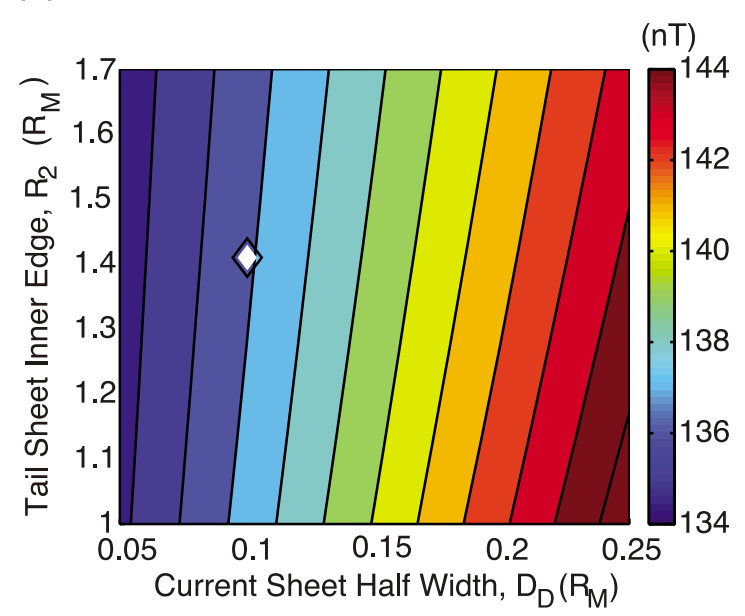

Figure 7. (a) Field magnitude, $B$, in the southern tail lobe for observations for which the field direction is within $10^{\circ}$ of antisunward. (b) Flux, $F$, as a function of distance downtail calculated from observations in Figure 7a and the Shue et al. [1997] magnetopause model. The mean flux is $2.6 \pm 0.6 \mathrm{MWb}$. (c) Sensitivity of the parameter $B_{\mathrm{T}}$ to $R_{2}$ and $D_{\mathrm{D}}$, calculated from the mean flux in Figure $7 \mathrm{~b}$ and equation (4) in the text. Diamond indicates mean $B_{\mathrm{T}}$ parameter calculated from our best estimates for $D_{\mathrm{D}}, R_{2}$, and $F$.

an excellent overall fit to the entire population of magnetopause crossings.

\subsection{Tail Current Sheet Geometry and Tail Flux}

[21] Orbits that enter the magnetosphere close to the midnight meridian provide information on the thickness and position of the current sheet in the deep-tail region (e.g., Figure 2), whereas orbits that enter the magnetosphere on the dayside close to noon local time sample the near-tail region during the descending portion of the transit. We used orbits from the deep tail to constrain the current sheet half thickness $\left(D_{\mathrm{D}}\right)$ and the tail flux $(F)$. We found that most orbits that sample the near-tail region do not typically cross the current sheet, and so these orbits provide only a bound on how close, on average, the current sheet comes to the planet $\left(R_{2}\right)$.

[22] For the deep-tail observations the current sheet was indicated by a rotation in the field direction from dominantly antisunward in the southern tail lobe to dominantly sunward in the northern tail lobe. The rotation can be seen on individual profiles (Figure 2), and we obtained a mean thickness for the current sheet in this region from a superposed epoch analysis. Orbits from the first three Mercury years of observations, with magnetic equator crossings within $3 \mathrm{~h}$ of midnight in the deep tail, were selected. The magnetic equator crossing for each orbit was assigned as the reference time, and the tilt of the field in the $\rho-Z$ plane was calculated 
(a)

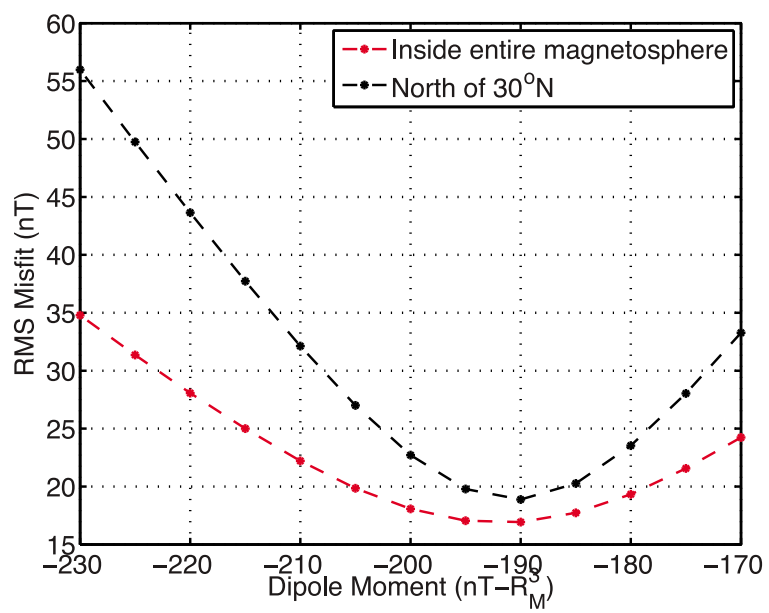

(b)

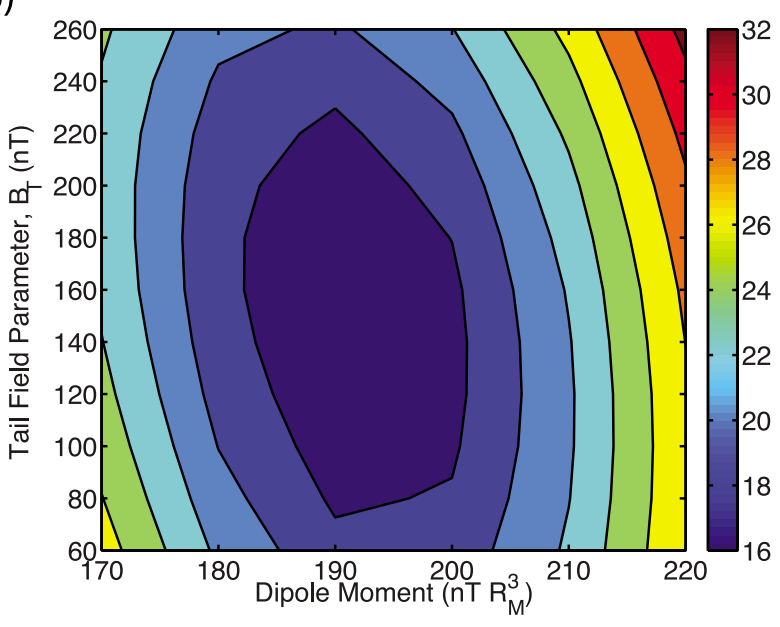

Figure 8. RMS misfit of predicted to observed $B$. (a) Misfit as a function of dipole moment for observations inside the entire magnetosphere (red curve) and for observations restricted to latitudes north of $30^{\circ} \mathrm{N}$ (all other model parameters are as specified in Table 1). (b) Misfit as a function of both dipole moment and tail field parameter $B_{\mathrm{T}}$.

from $\theta_{\rho-\mathrm{Z}}=\cos ^{-1}\left(B_{\rho} / B_{\rho \mathrm{Z}}\right)$, where $B_{\rho \mathrm{Z}}$ is the magnitude of $\mathbf{B}$ projected onto the $\rho-Z$ plane. Since $B_{\rho}$ is dominated by $B_{\mathrm{X}}$ in this region, $\theta_{\rho-Z}$ close to $0^{\circ}$ and $180^{\circ}$ indicate antisunward and sunward field directions, respectively. Note that since the magnetic equator is taken to be the point where $B_{\rho}=0$, the center of the current sheet coincides with the magnetic equator by definition. The angle $\theta_{\rho-Z}$ and the magnetic field magnitude for the 79 selected deep tail orbits were averaged, and the results are shown in Figures $6 \mathrm{a}$ and $6 \mathrm{~b}$. The rotation of the field direction from the southern to northern lobe is clear (black curve). After removal of the dipole field (red curve) as given by the best fit dipole moment described in section 4.4, the field is almost purely antisunward in the southern lobe and sunward in the northern lobe. The field rotation is $99 \%$ complete within $\pm 220 \mathrm{~km}$ of the center of the current sheet or within $0.09 R_{\mathrm{M}}$. The rotation is $95 \%$ complete within $140 \mathrm{~km}\left(0.06 R_{\mathrm{M}}\right)$ of the current sheet center and approaches its asymptotic value at a distance of about $350 \mathrm{~km}\left(0.14 R_{\mathrm{M}}\right)$. The depression in field magnitude associated with the plasma sheet [Korth et al., 2011] is centered on the field reversal, although the magnetic depression is broader than the field rotation, indicating a greater average thickness of the plasma sheet than the current sheet. The minimum field magnitude occurs at the center of the current sheet. From these results we set the PMM parameter $D_{\mathrm{D}}=0.09 R_{\mathrm{M}}$, with a lower bound of 0.06 $R_{\mathrm{M}}$ and an upper bound of $0.14 R_{\mathrm{M}}$.

[23] The near-tail region was sampled by MESSENGER on orbits with the descending orbit leg on the nightside (within $3 \mathrm{~h}$ of midnight). The magnetic signatures were very different in this region. Inspection of individual near-tail orbits showed that although the magnetic equator can be reliably pinpointed, clear localized rotations in the field consistent with current sheet crossings were generally not observed. This observation indicates that these near-tail trajectories did not pass from the northern tail lobe to the southern, but rather the spacecraft generally traversed the equator planetward of the cross-tail current. Consequently, the MAG data allow us only to place bounds on $R_{2}$. Depressions in the field magnitude, indicating spacecraft encounters with the plasma sheet, occurred on some but not all crossings of the near-tail region. This pattern suggests that the plasma sheet and, hence, possibly, the current sheet were present for only a fraction of the near-tail passes. Because in the deep tail the plasma sheet correlated with the current sheet, we selected near-tail orbits with stronger plasma sheet signatures by evaluating the minimum value of the magnetic field strength near the equator crossing. Those orbits with the strongest plasma sheet signatures and deepest magnetic field minima should be those that passed closest to the current sheet.

[24] Superposed epoch averages were obtained for the 25\% of the orbits with the lowest minimum field magnitudes (Figure 6c). We then subtracted the vector dipole field to assess the field properties due to external currents only, and we took the averages of the residual field magnitude, $Z$ component, and polar angle: $B, B_{\mathrm{Z}}$ and $\theta_{\rho-\mathrm{Z}}$.

[25] The near-tail field, even for these orbits, was quite different from the far-tail field. First, $\theta_{\rho-Z}$ came only within about $30^{\circ}$ of the $180^{\circ}$ or $0^{\circ}$ direction, indicating that the orbits were, on average, planetward of the current sheet and tail lobes. Second, the magnitude of the external field (after removal of the dipole field) increased rather than decreased near the equator crossing and at the equator was almost entirely in the $-B_{\mathrm{Z}}$ direction, that is, southward. This behavior indicates that the external field in this region was dominated by the fringing field of the cross-tail current planetward of the tail current sheet. Thus, these orbits passed close to but not through the current sheet, so we estimated a lower bound on $R_{2}$ from the mean radial distance $\left(\sqrt{X^{2}+Y^{2}}\right)$ to these equator crossings. On average $R_{2}$ was tailward of $1.41 R_{\mathrm{M}}$, and we set $R_{2}=1.41 R_{\mathrm{M}}$ in the PMM. The standard deviation in $R_{2}$ is $0.05 R_{\mathrm{M}}$, and we use this uncertainty to set upper and lower bounds on the mean value of $R_{2}$ (rather than the standard error in the mean) because the spacecraft orbit varied systematically in this region. We note that temporal variability of this distance is likely to occur on timescales less than one orbital period [e.g., Slavin et al., 2012]. In addition, because on average MESSENGER's 
(a) Model Predictions
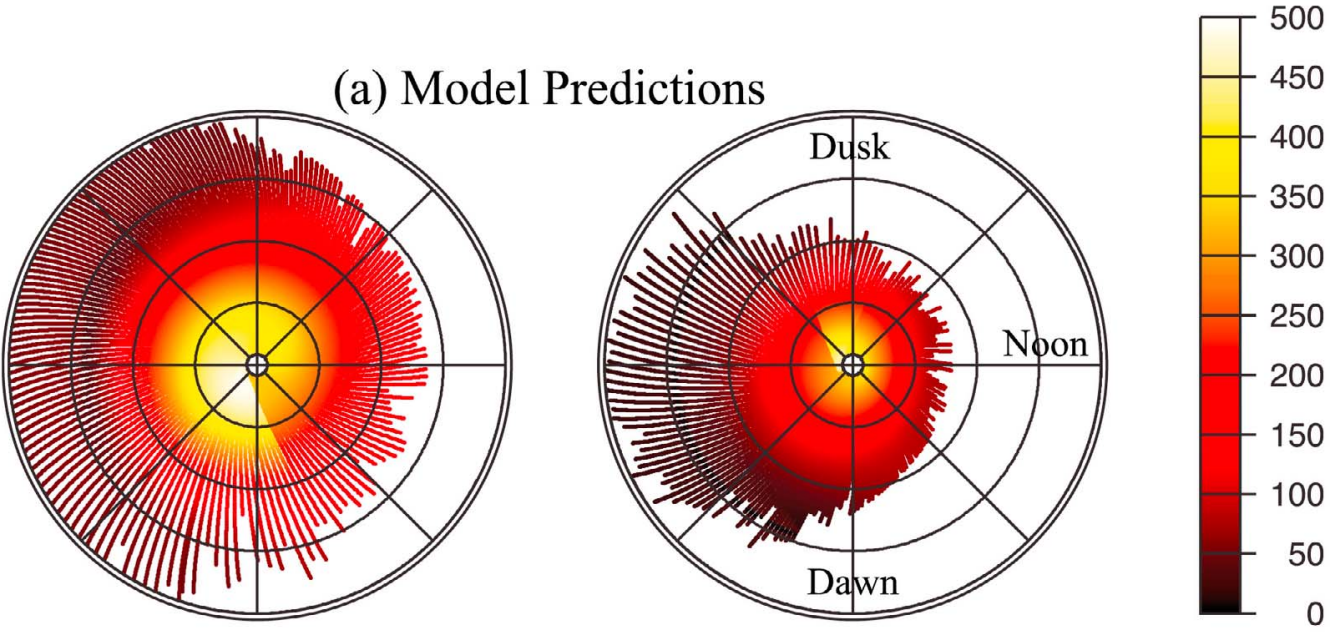

(nT)

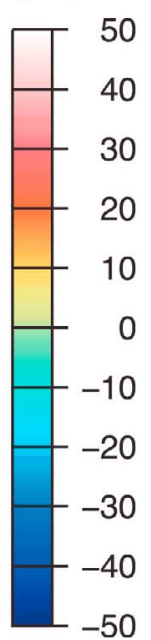

Figure 9. Stereographic projections in aberrated MSO coordinates of (a) predicted magnetic field magnitude, $B$, and (b) the corresponding residuals, where $B_{\text {resid }}=B_{\text {data }}-B_{\text {model }}$. Results are shown within the model magnetosphere, or within the actual magnetosphere if the observed magnetopause boundaries occurred within the model magnetopause boundary. (left) Descending and (right) ascending tracks from the second Mercury year of observations (20 June to 15 September 2011) are shown. Format as in Figure 3; grid lines are every $30^{\circ}$ in latitude $\left(30^{\circ} \mathrm{S}\right.$ to north pole) and every $3 \mathrm{~h}$ in local time.

orbit does not cross the current sheet in the near-tail region, we do not have an estimate of the current sheet half width close to the planet. We expect that $D_{\mathrm{D}}$ will be larger in the near-tail region than the far-tail region, but in the absence of near-tail current sheet crossings, we use our far-tail estimate of $D_{\mathrm{D}}$ in the PMM (Table 1).

[26] We estimated the tail flux parameter of the PMM model, $B_{\mathrm{T}}$, as follows. To ensure spacecraft residence in the lobe field, we selected only those observations in the southern tail for which the field was oriented to within $10^{\circ}$ of the antisunward direction. These locations and the corresponding field magnitudes at each point are shown in Figure 7a. At each location we used the field magnitude, $B$, together with the radius to the magnetopause boundary from the Shue et al. [1997] model, $r_{M P}$, measured from the $X_{\mathrm{MSM}}$ axis (to account for the offset dipole) parallel to the $Y_{\mathrm{MSO}}-Z_{\mathrm{MSO}}$ plane at the $X_{\mathrm{MSO}}$ coordinate of the spacecraft. We then estimated the southern lobe magnetic flux (Figure 7b) for each data point from

$$
F=B \pi r_{\mathrm{MP}}^{2} / 2 .
$$

The average tail flux is $2.6 \mathrm{MWb}$, and the standard deviation is $0.6 \mathrm{MWb}( \pm 25 \%)$. The tail flux was calculated from data obtained during 63 orbits, and so the standard error of the mean is $0.08 \mathrm{MWb}$. We used three standard errors to give the upper and lower values of the mean flux (Table 1). With this value of $F$, together with our estimates for $D_{\mathrm{D}}$ and $R_{2}$, we calculated the tail field parameter $B_{\mathrm{T}}$ in the PMM. Note that $B_{\mathrm{T}}$ depends on all the other parameters earlier estimated, including $\gamma, R_{1}, R_{2}$, and $D_{\mathrm{D}}$. Of these, $R_{2}$ is the least well constrained in an average sense, but for a given flux, $B_{\mathrm{T}}$ is relatively insensitive to both $D_{\mathrm{D}}$ and $R_{2}$. Figure $7 \mathrm{c}$ shows that for our mean flux, $B_{\mathrm{T}}$ varies by less than $6 \%$ for $D_{\mathrm{D}}$ values 

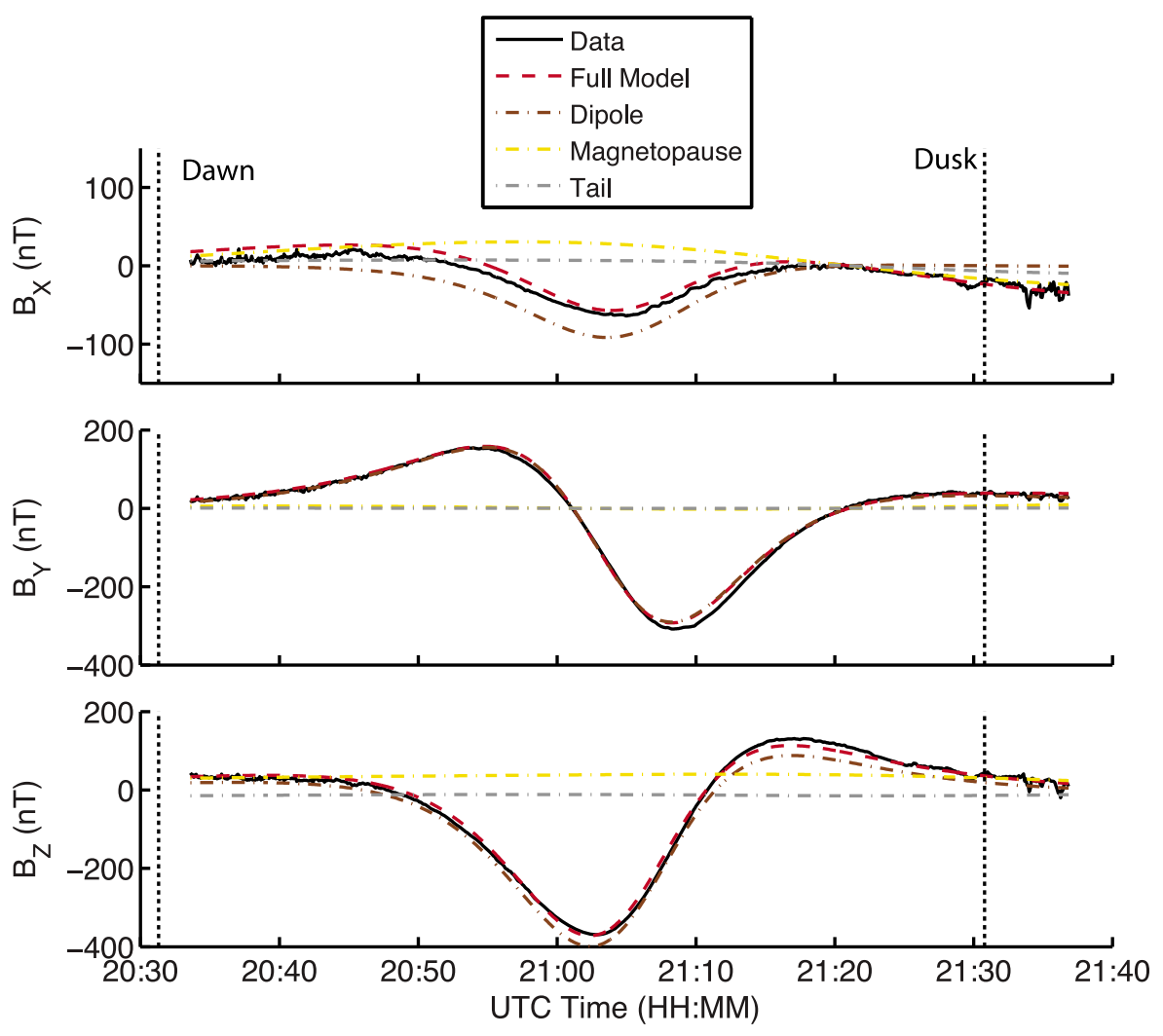

Figure 10. (top) Aberrated MSO $B_{\mathrm{X}}$, (middle) $B_{\mathrm{Y}}$, and (bottom) $B_{\mathrm{Z}}$ components of the magnetic field for the dawn-dusk orbit (orbit 274) on 1 August 2011 (from Figure 1), shown inside the model magnetopause, plotted at $5 \mathrm{~s}$ intervals. Time axis is UTC. For reference, the local dawnside and duskside (local time) of the orbit are labeled. Refer to Figure 1 for corresponding spacecraft latitude and altitude. For each magnetic field component, the data (black), full model prediction (red), dipole field (brown), magnetopause field (gold), and tail field (gray) are shown. Note the different vertical scale for $B_{\mathrm{X}}$. Vertical black dotted lines show the locations of the observed inner magnetopause crossing for this orbit. The RMS misfits of the full model to the $B_{\mathrm{X}}, B_{\mathrm{Y}}$, and $B_{\mathrm{Z}}$ data are $9.3 \mathrm{nT}, 8.7 \mathrm{nT}$, and $10.4 \mathrm{nT}$, respectively.

between $0.05 R_{\mathrm{M}}$ and $0.25 R_{\mathrm{M}}$, and for $R_{2}$ values between 1.0 $R_{\mathrm{M}}$ (the surface of Mercury) and $2.0 R_{\mathrm{M}}$. $B_{\mathrm{T}}$ scales linearly with the tail lobe flux, which varies from one orbit to another, reflecting different states of the magnetotail. Although the range in the mean flux is small $(0.2 \mathrm{MWb})$, instantaneous tail flux estimates range from 1 to $4.5 \mathrm{MWb}$, and in section 5 we verify that even a factor of 4 variation in the tail flux or the corresponding $B_{\mathrm{T}}$ values does not affect our estimates of the mean dipole moment.

\subsection{Reconnection Efficiency}

[27] We set the reconnection efficiency, $k$, to zero for our average baseline magnetospheric model. As the effect of reconnection is modeled simply by adding $k \mathbf{B}_{I M F}(0 \leq k \leq 1)$ to the predicted fields inside the magnetosphere, reconnection can be examined as one of the possible contributors to the residuals to the baseline model. Reconnection is likely to vary markedly from one orbit to the next and even during a given orbit. The IMF $B_{Z}$ component plays a critical role in reconnection, but at Mercury IMF $B_{Z}$ is small relative to IMF $B_{\mathrm{X}}$ and IMF $B_{\mathrm{Y}}$, and it varies on timescales much shorter than a magnetospheric transit time. When MESSENGER is inside the magnetosphere, we do not have simultaneous measurements of the IMF, and so it is unclear how best to estimate the factor $k \mathbf{B}_{\mathrm{IMF}}$ a priori, even on an orbit-by-orbit basis.

\subsection{Dipole Moment}

[28] We use the values of the PMM parameters estimated as described above as a priori constraints in the paraboloid model. The last remaining model parameter, the dipole moment, $m$, is constrained by the goodness of fit of the model to the MAG data, globally. We describe the fit process and the resulting global model fields in section 5 .

\section{Global Magnetospheric Fields: Results and Discussion}

[29] The mean values of the magnetospheric parameters estimated by the approaches described above are summarized in Table 1. To complete the PMM model, we conducted a grid search to determine the best fit dipole moment to the observations in an RMS sense. The dipole moment was varied from 170 to $230 \mathrm{nT} R_{\mathrm{M}}^{3}$ in $5 \mathrm{nT} R_{\mathrm{M}}^{3}$ steps. For each value of the dipole moment, the magnetopause, magnetotail, and dipole fields were predicted at $5 \mathrm{~s}$ intervals along every orbit track inside the model magnetopause. The model was compared with the data in the $5 \mathrm{~s}$ RDRs, and the 

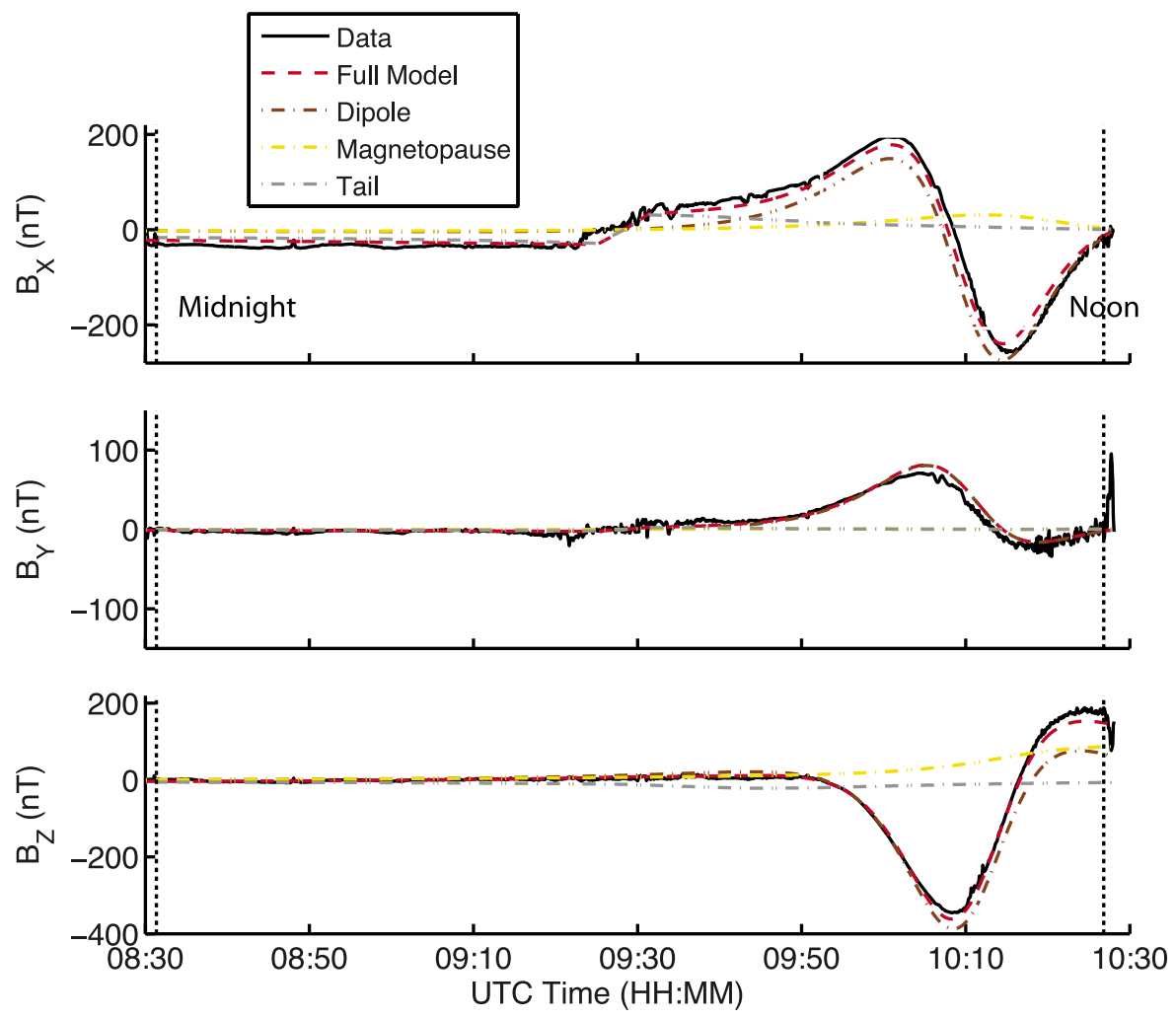

Figure 11. (top) Aberrated MSO $B_{\mathrm{X}}$, (middle) $B_{\mathrm{Y}}$, and (bottom) $B_{\mathrm{Z}}$ components of the magnetic field for the midnight-noon orbit (orbit 497) on 20 November 2011 (from Figure 1), shown inside the model magnetopause, plotted at $5 \mathrm{~s}$ intervals. Format as in Figure 10. Time axis is UTC. The midnight and noon (local time) sides of the orbit are labeled. Note the different vertical scale for each component. The RMS misfits of the full model to the $B_{\mathrm{X}}, B_{\mathrm{Y}}$, and $B_{\mathrm{Z}}$ data are $15.3 \mathrm{nT}, 7.4 \mathrm{nT}$, and $19.4 \mathrm{nT}$, respectively.

RMS misfit of the predicted field to the observations was computed (Figure 8). Both globally inside the entire magnetosphere and for the region restricted to latitudes north of $30^{\circ} \mathrm{N}$ (where the dipole field dominates), a best fit dipole moment of $190 \mathrm{nT} R_{\mathrm{M}}^{3}$ was found. For the latter region, the minimum misfit is $18.9 \mathrm{nT}$, and the RMS misfit is within $20 \%$ of this minimum value for dipole moments within $\pm 10 \mathrm{nT} R_{\mathrm{M}}^{3}$ of $190 \mathrm{nT} R_{\mathrm{M}}^{3}$.

[30] Among the PMM parameters estimated from the observations, $B_{\mathrm{T}}$ likely deviates the most from its average value on an orbit-by-orbit basis (section 4.3). A second grid search confirms that the best fit dipole moment is insensitive to the value of $B_{\mathrm{T}}$ for $B_{\mathrm{T}}$ values in the range 60 to $260 \mathrm{nT}$ (Figure $8 \mathrm{~b}$ ), or equivalently the observed factor of 4 variations in tail flux (Figure 7b). Orbit-to-orbit variations in the tail flux result in corresponding orbit-to-orbit variations in the residuals to the baseline model in the tail region, as discussed further in section 6 , but have negligible influence on the planetary moment estimate. We also verified that the best fit dipole moment is insensitive, at the level of the $5 \mathrm{nT}$ $R_{\mathrm{M}}^{3}$ increment used in the grid search, to changes in the other parameters within the range of mean values given in Table 1. The robustness of the fit illustrates the advantage of the approach taken here: that of first estimating as many magnetospheric model parameters as possible directly from the observations, and then using these parameters as a priori constraints in the paraboloid model. The alternative approach, that of coestimating all model parameters from nonlinear inversions of the vector data inside the magnetosphere, would yield trade-offs among model parameters, in particular among the dipole moment, the dipole offset, the magnetopause shape, and the distance from the dipole origin to the magnetopause subsolar point. Furthermore, such an approach would ignore robust a priori observational constraints on model parameters.

[31] Comparison of the predicted model field magnitudes along the orbit tracks for the second year of MESSENGER observations (Figure 9) with the corresponding MAG observations (Figure 3 ) demonstrates the overall success of the model. Residuals (data minus model) are typically less than $50 \mathrm{nT}$ in magnitude (Figure 9) compared with a maximum signal in the data of $\sim 500 \mathrm{nT}$. Model predictions for the aberrated MSO $B_{\mathrm{X}}, B_{\mathrm{Y}}$, and $B_{\mathrm{Z}}$ components for our example dawn-dusk and midnight-noon orbits provide an excellent fit to the observations (Figures 10 and 11). In addition to the full model prediction, the individual model tail, magnetopause, and dipole fields are shown along each orbit. The importance of the magnetopause fields at high northern latitudes and on the dayside can be seen. For orbit 274 on 1 August 2011 (Figure 10), the dipole field alone would overpredict the amplitudes of the $B_{\mathrm{X}}$ and $B_{\mathrm{Z}}$ components of the field by $\sim 40 \%$ and $\sim 10 \%$, respectively. For orbit 497 on 20 November 2011 (Figure 11), the dipole and magnetopause fields contribute almost equally to $B_{\mathrm{Z}}$ on the dayside. The magnetopause and dipole fields were essentially zero in the far tail for orbit 497 , and the negative $B_{\mathrm{X}}$ 
(a) Offset Dipole Field

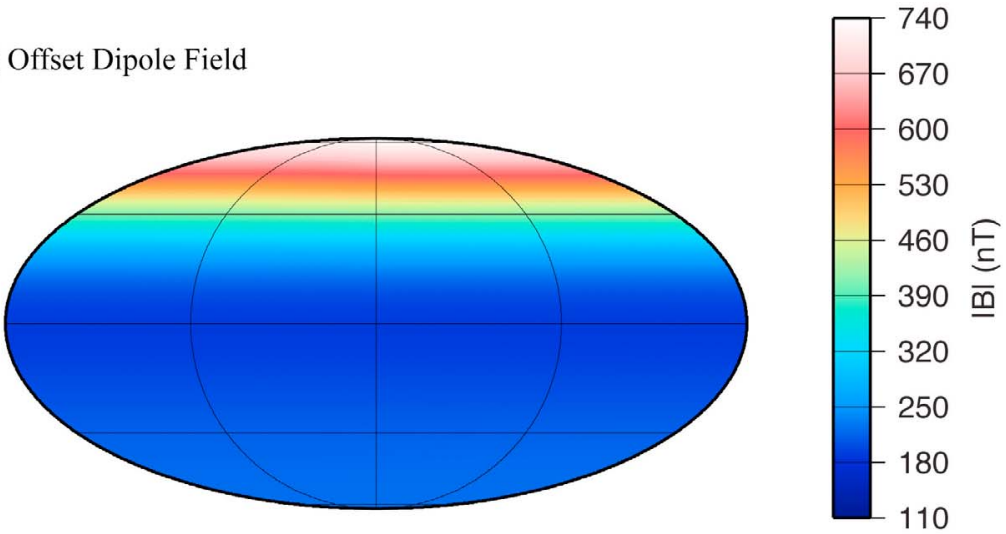

(b) Magnetopause Field
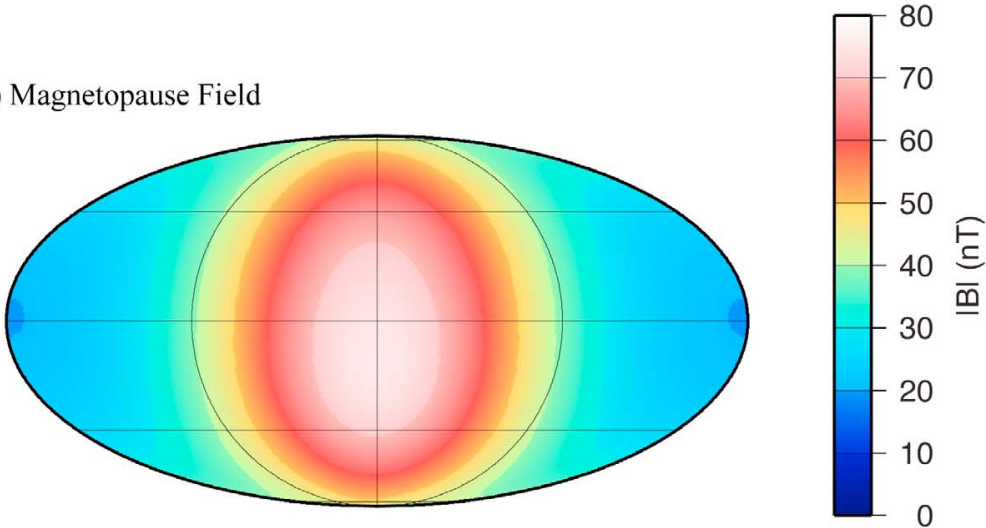

(c) Tail Field
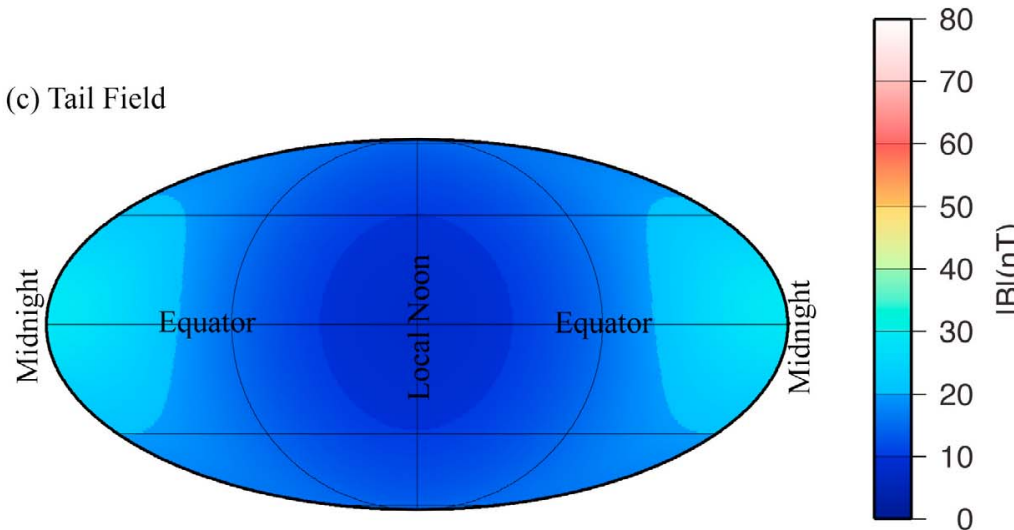

Figure 12. Mollweide projections of magnetic field magnitudes predicted at Mercury's surface $\left(R_{\mathrm{M}}=\right.$ $2440 \mathrm{~km}$ ) for the (a) offset dipole, (b) magnetopause, and (c) tail model fields. Plots are in MSO coordinates and are centered on local noon $\left(0^{\circ} \mathrm{MSO}\right.$ longitude) and $0^{\circ}$ latitude. Grid lines are every $45^{\circ}$ in latitude and every $90^{\circ}$ in MSO longitude ( $6 \mathrm{~h}$ in local time). Note the different scale bar for the external (magnetopause and tail) and internal (offset dipole) fields.

signal seen in the observations is predicted by the tail field component of the PMM.

[32] The magnitudes of the magnetopause, tail, and dipole fields predicted at the planetary surface are shown in Figure 12. The strong north-south asymmetry in the internal field imposed by the large northward offset of the dipole is evident, with the field strength at high northern latitudes over $700 \mathrm{nT}$, approximately three times that at the corresponding southern latitudes. Magnetopause fields are strongest on the dayside as expected. In addition, the offset dipole results in a magnetopause surface that is closer to the planetary surface at southern latitudes than at corresponding northern latitudes (see also Figure 4). This north-south difference is greatest at low to middle latitudes at local noon, and thus the strongest magnetopause fields are found at low southern dayside latitudes, where the magnetopause field strength reaches almost $80 \mathrm{nT}$, about $40 \%$ of the dipole field strength at those locations. 

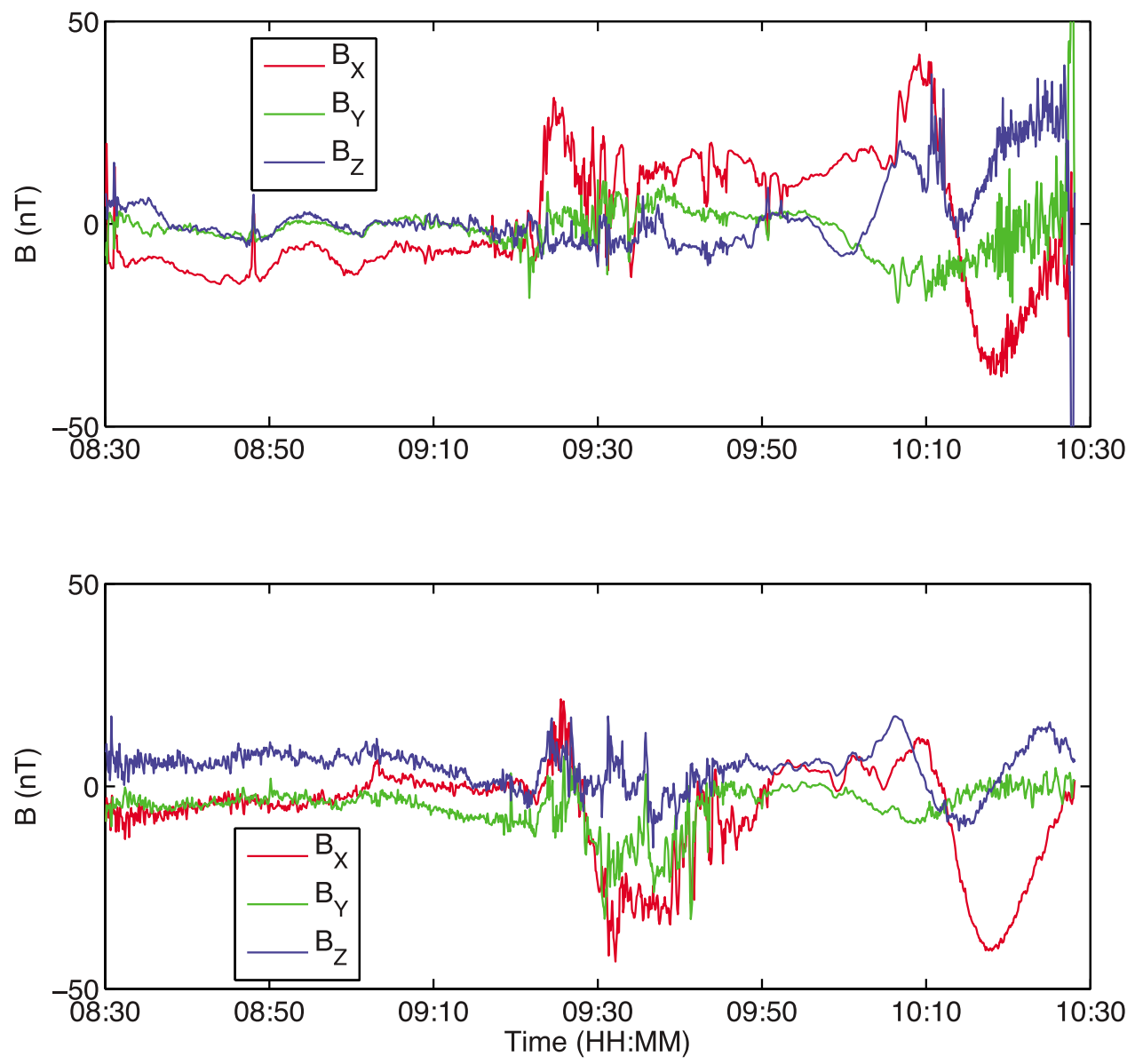

Figure 13. Residuals (data minus model in the aberrated MSO coordinate system) for (top) orbit 497 on 20 November 2011 and (bottom) orbit 499 on 21 November 2011. The two orbits are separated in time by $\sim 24 \mathrm{~h}$ and have similar ground tracks. The ascending portions of the tracks are close to midnight local time and at about $10^{\circ} \mathrm{MBF}$ longitude. Note the similar overall structure in the residuals but differences in the details, e.g., in $B_{\mathrm{X}}$ between 09:30 and 09:50 UTC, in the magnitude of the $B_{\mathrm{X}}$ and $B_{\mathrm{Z}}$ residuals in the tail, and in the high-frequency variability on the dayside and nightside.

[33] Temporal variations in Mercury's global-scale magnetic fields are expected to occur, due to the variation in solar wind ram pressure, $P_{\text {ram }}$, at Mercury that arises from changes in heliocentric distance during Mercury's orbit around the Sun. For a simple balance between the internal magnetic field pressure and the solar wind dynamic pressure, under the assumption of a constant planetary dipole moment, the magnetopause subsolar distance will vary as $P_{\mathrm{ram}}^{-1 / 6}[$ Shue et al., 1997]. Thus, if our mean $R_{\mathrm{SS}}$ value of $1.45 R_{\mathrm{M}}$ corresponds to average $P_{\text {ram }}$ conditions at Mercury, we can expect a variation in $P_{\text {ram }}$ about this average value of $\sim 50 \%$ and a corresponding variation in $R_{\mathrm{SS}}$ of about $\pm 7 \%$ or \pm 0.1 $R_{\mathrm{M}}$. This range is consistent with observed variations in $R_{\mathrm{SS}}$ (Winslow et al., submitted manuscript, 2012). The variations in $R_{\mathrm{SS}}$ result in changes in the magnitude of the magnetopause fields, in particular on the dayside. For $R_{\mathrm{SS}}$ variations of $\pm 0.1 R_{\mathrm{M}}$ about the mean value of $1.45 R_{\mathrm{M}}$, the magnetopause field magnitudes at spacecraft altitudes vary by $25 \%$ about their mean values on the dayside and on the nightside at latitudes north of $60^{\circ} \mathrm{N}$. In addition, shorter timescale variations in $R_{\mathrm{SS}}$ may occur in association with rapid, large- amplitude changes in $P_{\text {ram }}$ such as those due to coronal mass ejections or quasi-parallel bow shock dynamics.

\section{Residual Signatures: Results and Discussion}

[34] Residuals for each field component for midnightnoon orbits 497 and 499 on 20 and 21 November 2011 are shown in Figure 13. Orbit 499 occurred 24 h after orbit 497, so these orbits sampled the same large-scale static structure in the internal and external magnetic fields. There is some similar structure in the residuals for each orbit. Both orbits exhibit a large dayside negative residual in $B_{\mathrm{X}}$ (after 10:10 UTC), accompanied by a positive residual in $B_{\mathrm{Z}}$. However, differences in the residuals are also observed. For example, the large-scale structure in the $B_{\mathrm{X}}$ residuals is different between about 9:30 and 10:10 UTC, corresponding to northern nightside latitudes (Figure 2) near midnight local time and MBF longitude $\sim 10^{\circ}-15^{\circ} \mathrm{E}$. The magnetic field during orbit 497 was relatively quiet in the deep tail (08:30 09:10 UTC), whereas the field during orbit 499 was notably more disturbed as evidenced by the high-frequency 

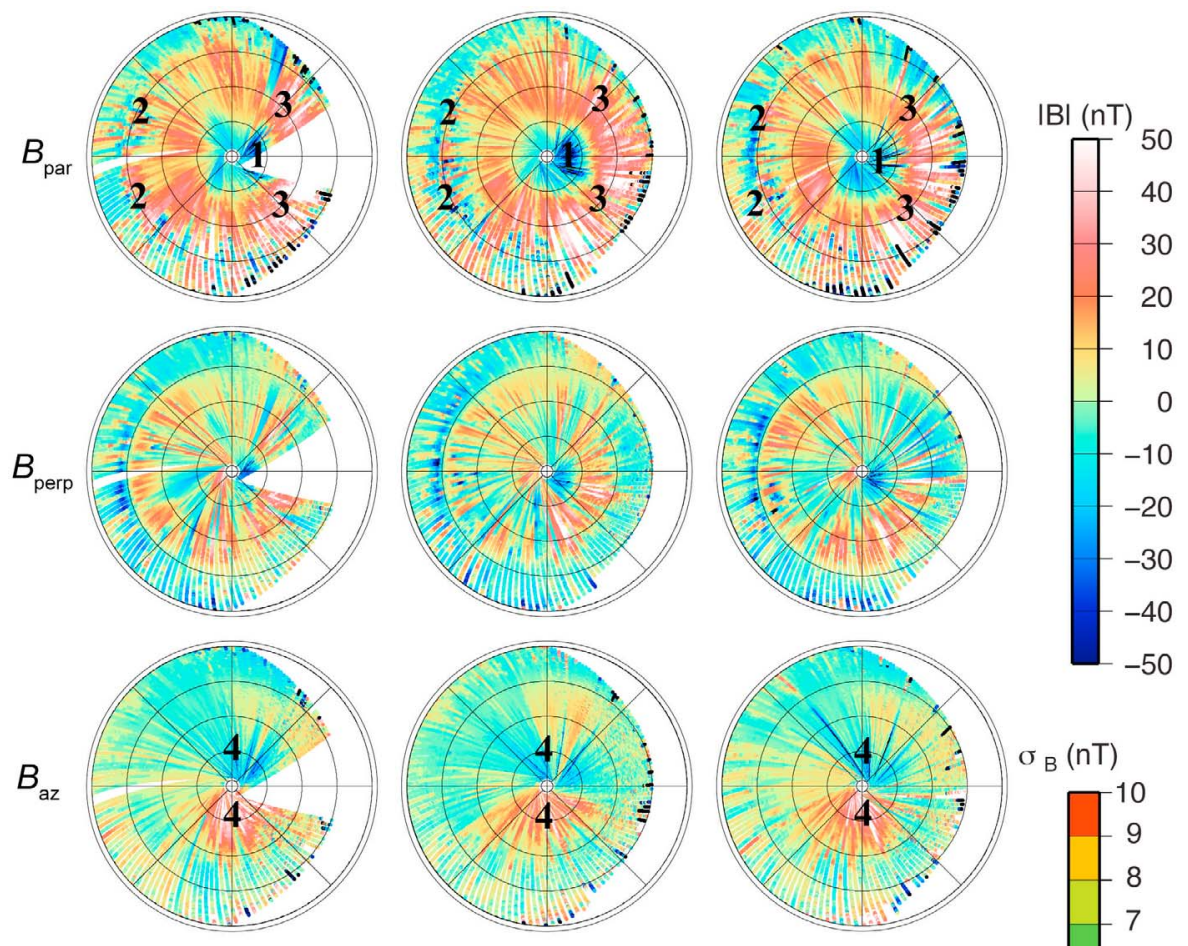

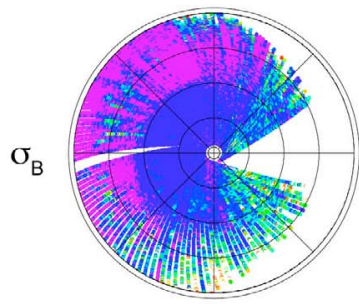

Days 83-170

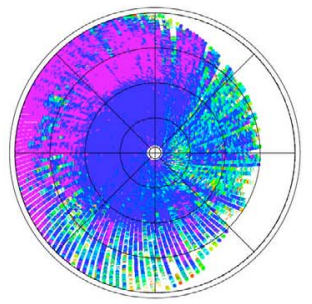

Days 171-258

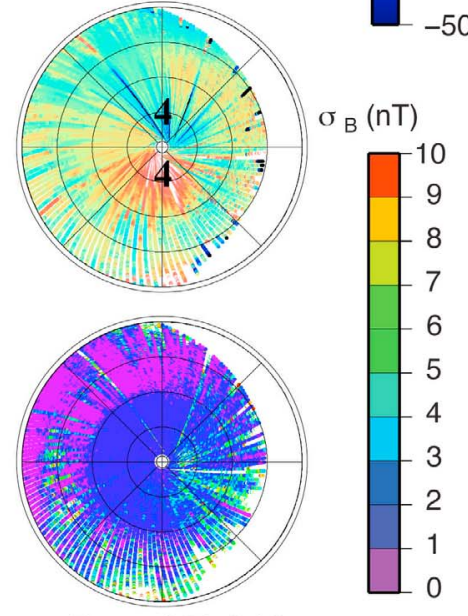

Days 259-346

Figure 14a. Stereographic projections in aberrated MSM coordinates of residuals and standard deviations over a $5 \mathrm{~s}$ interval in the total field $\left(\sigma_{\mathrm{B}}\right)$ for descending (lower altitude) orbit tracks for observations from Mercury (left) year 1, (middle) year 2, and (right) year 3 . Grid lines are every $30^{\circ}$ in latitude $\left(30^{\circ} \mathrm{S}\right.$ to $90^{\circ} \mathrm{N}$ magnetic latitude) and every $3 \mathrm{~h}$ in local time. Local noon is to the right. The first to third rows show the residuals rotated into the field-parallel $\left(B_{\mathrm{par}}\right)$ (left), principal normal $\left(B_{\text {perp }}\right)$ (middle), and azimuthal $\left(B_{\mathrm{az}}\right)$ (right) directions. Separate color scales are shown for the residuals and for the standard deviations. Regions labeled 1 through 4 are discussed in the text and are interpreted as (1) cusp (labeled only on $B_{\text {par }}$ ), (2) tail plasma sheet, and $(3,4)$ possible magnetopause signatures.

variability. Orbit 499 shows a persistent positive $B_{\mathrm{Z}}$ residual throughout most of the southern tail lobe until 09:10 UTC, or an MSO latitude of $\sim 10^{\circ} \mathrm{S}$. The $B_{\mathrm{X}}$ residuals from 09:30 to 09:40 UTC have opposite signs on the two orbits. On the dayside, the magnetic field during orbit 499 was relatively quiet and its inner magnetopause boundary was farther from the planet, compared with orbit 497. Even this cursory comparison of the residuals from these two orbits indicates that time-variable signatures can be comparable to the average residuals but suggests that some systematic residual signatures may be present.

[35] To examine the data for consistent signatures, we first considered the residuals in aberrated MSM coordinates. We transformed the residuals into coordinates organized by the baseline model magnetic field, where $B_{\mathrm{par}}$ is parallel to the model field direction, $\mathbf{n}_{\mathrm{par}}, B_{\mathrm{az}}$ is azimuthal and parallel to the $\phi_{\mathrm{MSO}}$ unit vector, $\mathbf{n}_{\phi}$, and $B_{\text {perp }}$ lies in the plane of the model magnetic field and the MSO $Z$ axis such that $\mathbf{n}_{\text {perp }}=$ $\mathbf{n}_{\phi} \times \mathbf{n}_{\mathrm{par}}$. Figure 14 shows the residuals in these coordinates for the first three Mercury years of observations. In $B_{\text {par }}$, diamagnetic depressions were seen consistently at dayside latitudes north of $60^{\circ} \mathrm{N}$ within $\pm 3 \mathrm{~h}$ of local noon on both the descending (Figure 14a) and ascending (Figure 14b) tracks, reflecting increased plasma pressure in the northern cusp region [Korth et al., 2011; Winslow et al., 2012]. Depressions in $B_{\mathrm{par}}$ associated with the tail plasma sheet were often seen around the deep-tail magnetic equator crossings (Figure 14b). Within about $1.5 \mathrm{~h}$ of local midnight, near-planet diamagnetic depressions were seen (Figure 14a); these variations reflect plasma sheet pressures as discussed by Korth et al. [2012]. Consideration of the plasma pressure implied by these near-tail magnetic field depressions shows that the plasma pressures are symmetric about the magnetic equator [Korth et al., 2012].

[36] Other major signals that were consistently present are positive residuals parallel to the local field direction at 

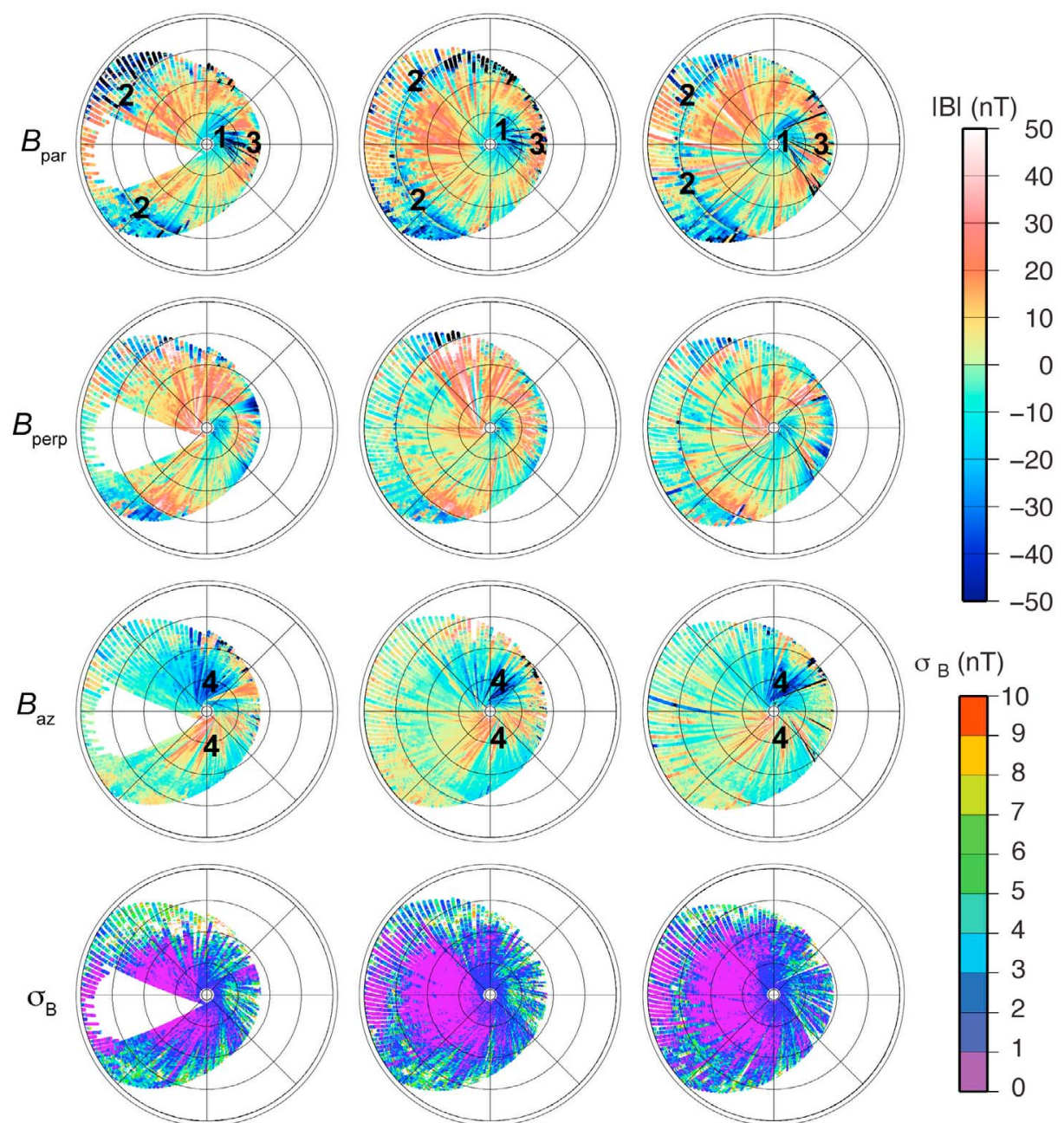

Days 83-170

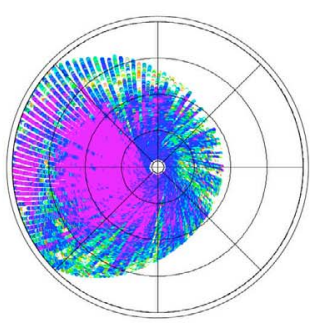

Days 171-258

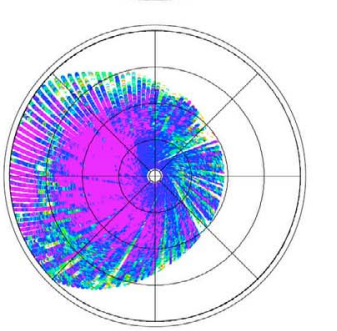

Days 259-346

Figure 14b. Same as Figure 14a but for the ascending (higher-altitude) portions of each orbit. Typical orbit altitudes for both ascending and descending tracks can be seen in Figure 3.

latitudes between $30^{\circ} \mathrm{N}$ and $60^{\circ} \mathrm{N}$ and positive (negative) dawn (dusk) residuals at high latitudes in the azimuthal $\left(B_{\mathrm{az}}\right)$ component. Note that the duskside $B_{\mathrm{az}}$ residual signature is also stronger in the higher-altitude data. We suggest that these signatures may in part be due to the difference between the actual magnetopause geometry and that from the paraboloid model. As seen in Figure 5, the paraboloid model magnetopause is more flared than the observations, in particular on the nightside. To assess the major signatures that could be affected by using a different magnetopause shape, we calculated the residuals with respect to a paraboloid magnetopause that is less flared than the $\gamma=1$ baseline model, corresponding to a smaller-diameter magnetotail. We specified a model magnetopause that is less flared, using $\gamma=$ 1.3 , chosen purely to illustrate the effect that such a magnetopause displacement would have. The $\gamma=1.3$ model provides a better fit to the magnetopause crossings on the nightside for $-0.5 R_{\mathrm{M}}<X_{\mathrm{MSO}}<2.0 R_{\mathrm{M}}$ but does not fit the dayside crossings as well as the $\gamma=1$ magnetopause shape. Residual signatures larger than $10 \mathrm{nT}$ amplitude are shown in Figure 15 for both our baseline model and the $\gamma=1.3$ model for the second Mercury year of observations, together with the difference between the $\gamma=1.0$ and $\gamma=1.3$ models. The experiment indicates that some, but not all, of the structure in the residual signatures in the $B_{\text {par }}$ and $B_{\text {perp }}$ components is correlated with structure introduced, or removed, by changing the magnetopause shape. Although signatures similar to those observed in the $B_{\mathrm{az}}$ component result from flattening the magnetopause shape, their amplitude is less than $10 \mathrm{nT}$, and these signatures in the residuals are thus of a different origin.

[37] Steady, nondipolar internal field signatures (of either core or crustal origin) should exhibit residual signatures in the MBF frame that are consistent from one sidereal day to the next. The vector residuals for four sidereal days, starting on 24 March 2011 in MBF spherical coordinates $\left(B_{\mathrm{r}}, B_{\theta}\right.$, $B_{\phi}$ ), are shown in Figure 16 for descending (lower altitude) tracks only, for latitudes north of $30^{\circ} \mathrm{N}$ (i.e., where spacecraft altitudes are below $1000 \mathrm{~km}$ ). Signatures such as the cusp region, which are well organized in the MSO frame, are seen rotated in the MBF frame as positive signatures in both $B_{\mathrm{r}}$ and $B_{\theta}$. These signatures occur at similar MBF longitudes every third sidereal day because of Mercury's 3:2 spin orbit resonance. A positive anomaly in $B_{\mathrm{r}}$ at latitudes north of 
(a) Residuals relative to $\gamma=1$ model
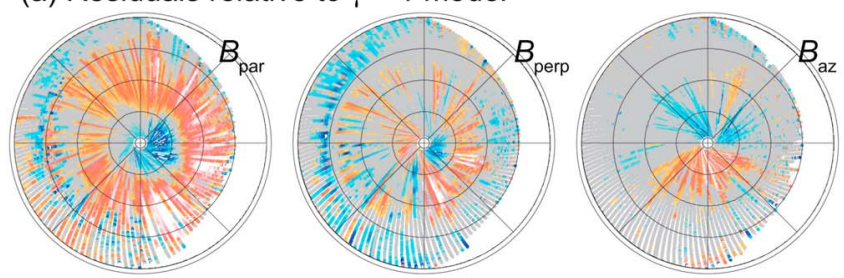

(b) Residuals relative to $\gamma=1.3$ model
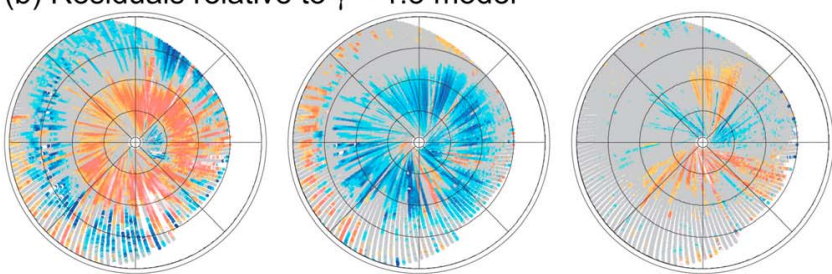

(c) $\gamma=1.3$ model minus $\gamma=1$ model
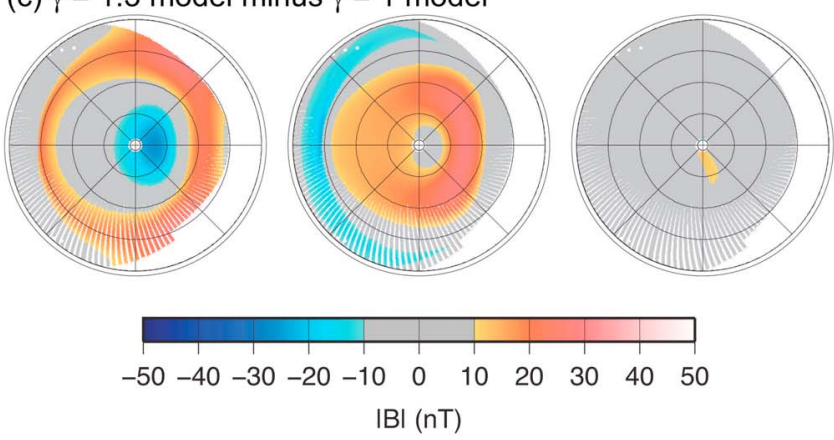

Figure 15. Residuals in aberrated MSM coordinates relative to a model with magnetopause flaring (a) $\gamma=1.0$ and (b) $\gamma=1.3$ (all other parameters as in Table 1). (c) Difference between the models with $\gamma=1.3$ and $\gamma=1.0$. The (left) $B_{\text {par }}$, (middle) $B_{\text {perp }}$, and (right) $B_{\text {az }}$ components are shown. Moving the magnetopause closer to the planet (less flaring) results in increased fields in the $B_{\text {par }}$ component at low to middle latitudes and decreased $B_{\text {par }}$ at latitudes north of $60^{\circ} \mathrm{N}$. The latter signature results in a change in the magnitude of $B_{\mathrm{r}}$ at latitudes north of $60^{\circ} \mathrm{N}$ in the MBF frame.

$60^{\circ} \mathrm{N}$, and elongated approximately along $45^{\circ} \mathrm{E}$ longitude, is seen in all four sidereal days. In contrast, large signals (up to $50 \mathrm{nT}$ amplitude) are seen in the $B_{\theta}$ and $B_{\phi}$ components that are not consistent from one sidereal day to the next. These signatures are better organized in the MSO frame, suggesting an external origin. In a separate study, Purucker et al. [2012] address the question of a possible internal origin for the MBF signature in $B_{\mathrm{r}}$ has been addressed.

[38] The residuals to our baseline model in both MSO and MBF coordinates often show large orbit-to-orbit variations. Such variations, as well as changes in the residuals on timescales shorter than MESSENGER's orbital period, likely reflect a combination of processes. These include variations in $R_{\mathrm{SS}}$ (driven by short timescale changes in $P_{\text {ram }}$ ), as well as possible changes in magnetopause flaring $(\gamma)$, tail flux $(F)$, and tail current sheet parameters $D_{\mathrm{D}}$ and $R_{2}$ associated with reconnection. As discussed earlier, we set the reconnection efficiency, $k$, in the PMM model to zero in our baseline model. However, observations of the magnetic shear across the dayside magnetopause suggest that the average reconnection efficiency may be substantial (Winslow et al., submitted manuscript, 2012), as expected at Mercury [Slavin et al., 2010, 2012]. Residual signatures also vary markedly in their high-frequency content (e.g., Figure 13). Investigations of higher-order structure in the internal field should be confined to periods when such high-frequency contributions are absent.

\section{Conclusions}

[39] Vector magnetic field observations acquired by MESSENGER in orbit around Mercury have allowed, for the first time, robust identification of the planet's globalscale magnetospheric fields. We used observations taken over three Mercury years and 4.5 sidereal days, spanning the period 24 March to 12 December 2011. These measurements provide repeated complete coverage over Mercury's northern hemisphere in local time and in body-fixed longitude, as well as sampling of Mercury's southern magnetotail region. The repeated coverage has permitted investigations of timeaveraged signatures in the global-scale fields and allowed identification of both steady and time-varying signatures in the residuals to the global fields.

[40] We modeled the large-scale external fields from magnetopause and magnetotail currents and the internal dipole field with a paraboloid magnetospheric model [Alexeev and Shabansky, 1972; Belenkaya et al., 2005; Alexeev et al., 2008, 2010]. All but one of the model parameters are specified by direct observations of the magnetopause shape, the tail current sheet geometry, the tail flux, and the dipole origin and tilt. For each MESSENGER orbit we identified the locations of the inbound and outbound magnetopause crossings (Winslow et al., submitted manuscript, 2012). We used these crossing positions to determine the average shape of the magnetopause boundary, assumed for the modeling to be a paraboloid of revolution. The magnetopause crossings indicate an average subsolar standoff distance, $R_{\mathrm{SS}}$ of $1.45 R_{\mathrm{M}}$, and, on the dayside and near-planet nightside, are consistent with a flaring parameter $\gamma=1$. The orientation and origin of the internal dipole field relative to the planetary center were obtained through identification of the magnetic equator on nearly every orbit [Anderson et al., 2011, 2012], excepting only those orbits on which MESSENGER exited the magnetosphere on the dayside before crossing the magnetic equator. The dipole origin is offset northward from the planet center by $0.196 R_{\mathrm{M}}$, and to a good approximation the dipole is aligned with the rotation axis. Because the dipole offset from the planet center is so large, the equivalent spherical harmonic description of the field in the MBF frame requires not only an axial quadrupole term that is $40 \%$ of the axial dipole term at the planetary surface, but also contributions from axial terms of degree 3 and 4 that are $12 \%$ and $3 \%$ of the axial dipole term, respectively [see Anderson et al., 2012]. Magnetic field strengths observed in the southern tail lobe, together with a Shue et al. [1997] model for the magnetopause shape, indicate a mean flux in one tail lobe of $2.6 \mathrm{MWb}$, with a standard deviation of $\sim 25 \%$ of this mean value. Crossings of the tail current sheet indicate a mean half width for the current sheet of $0.1 R_{\mathrm{M}}$ in the deep tail. The absence of current sheet crossings in the near-tail region suggests that the closest approach of the current sheet to the planet is on average $1.41 R_{\mathrm{M}}$ or greater. The best fit dipole moment was obtained via a grid search 

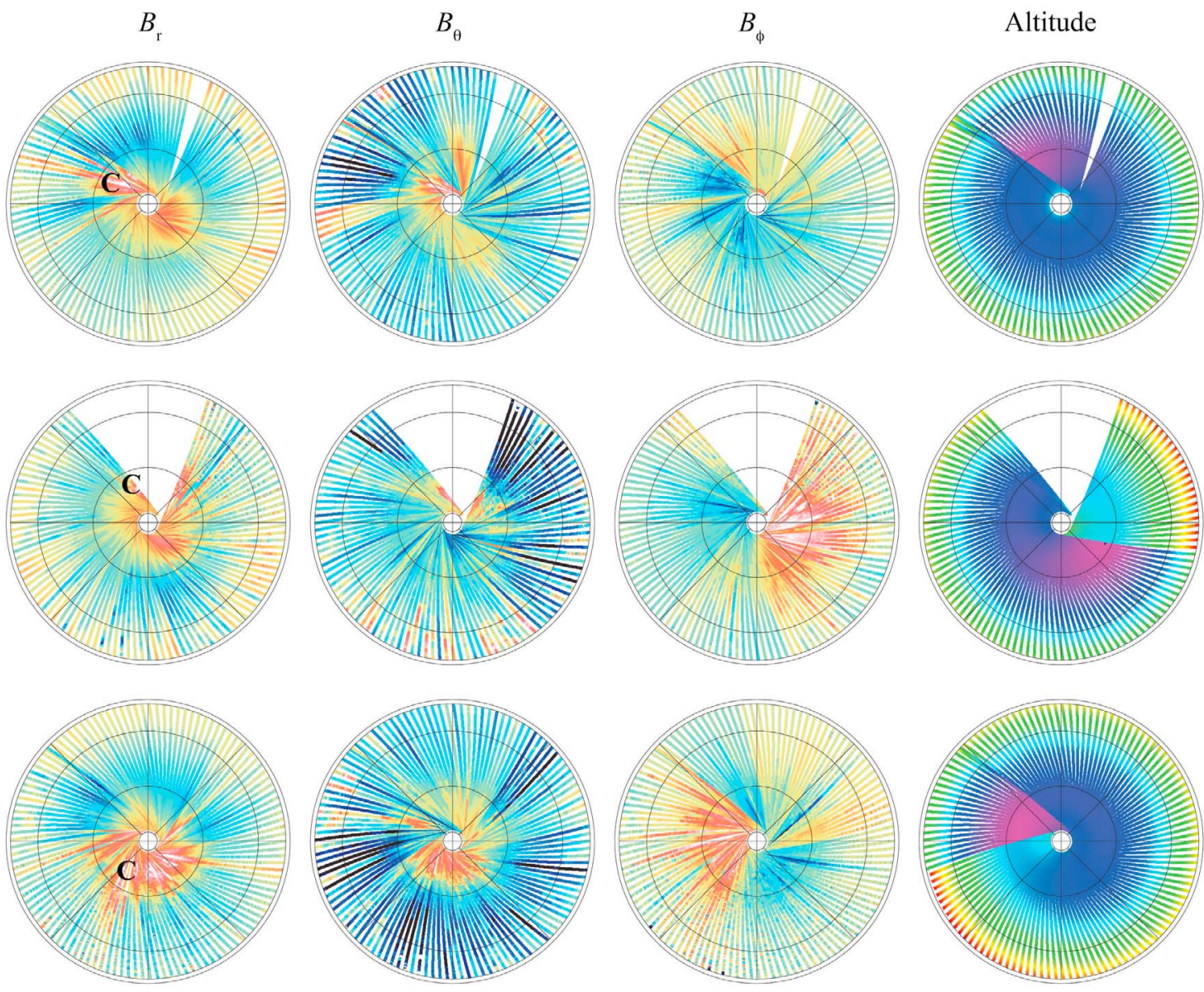

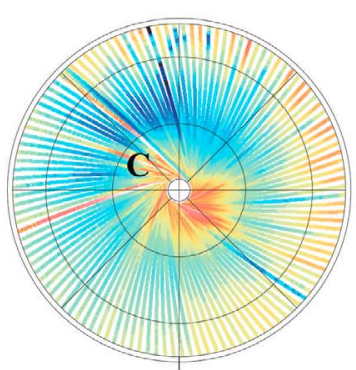

$0^{\circ}$

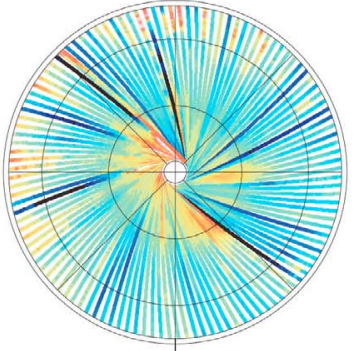

$0^{\circ}$

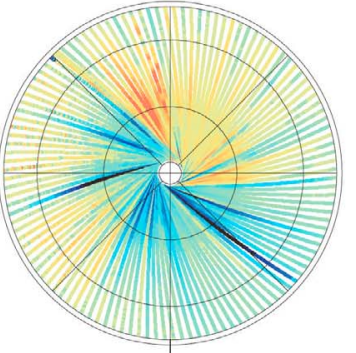

$0^{\circ}$
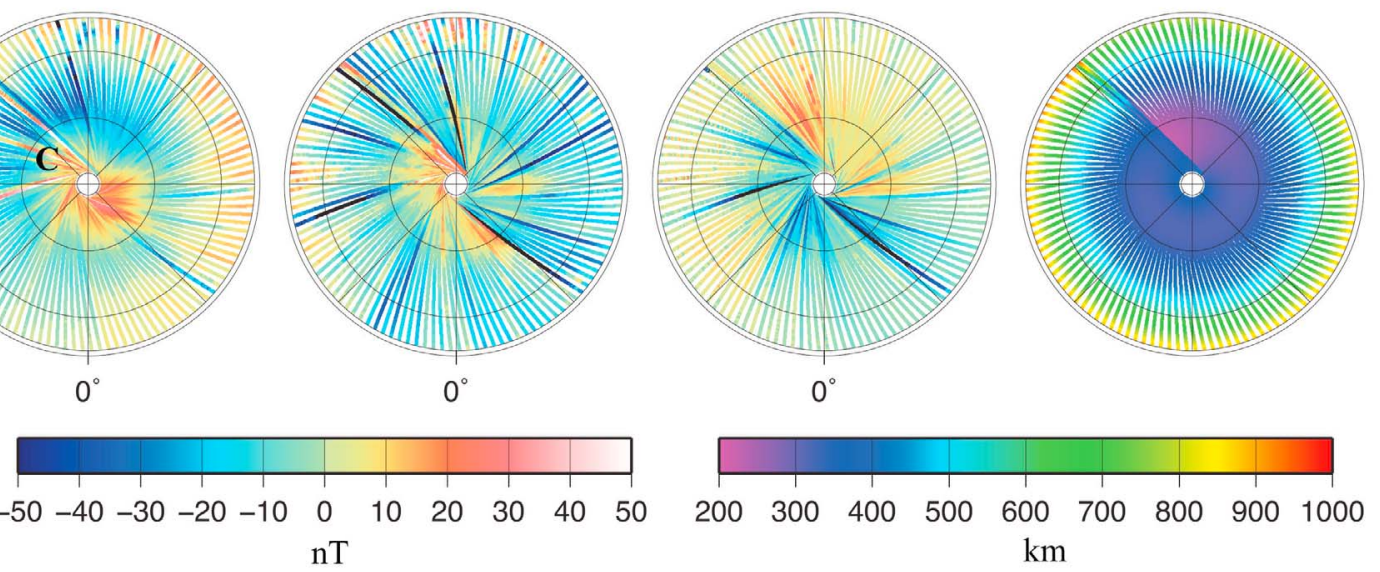

Figure 16. Residuals in MBF format for the first four coverages (59 Earth days each) of body-fixed longitude (first to fourth rows). The (left) $B_{\mathrm{r}}$, (middle) $B_{\theta}$, and (right) $B_{\phi}$ residuals in nT (left color bar at bottom) and spacecraft altitude in $\mathrm{km}$ (right color bar at bottom) are shown. Structure in the spacecraft altitude figures reflects the drift of periapsis altitude to higher elevations and the periapsis-lowering maneuvers that occurred every 88 days. $0^{\circ} \mathrm{MBF}$ longitude is toward bottom of each plot; plots show latitudes only northward of $15^{\circ} \mathrm{N}$; grid lines are at $30^{\circ} \mathrm{N}, 60^{\circ} \mathrm{N}$, and at $45^{\circ}$ longitude intervals. Region labeled " $C$ " denotes cusp.

and found to be $190 \mathrm{nT} R_{\mathrm{M}}^{3}$. Dipole moments within $10 \mathrm{nT}$ $R_{\mathrm{M}}^{3}$ of the $190 \mathrm{nT} R_{\mathrm{M}}^{3}$ best fit value yield less than a $20 \%$ increase in RMS misfit, and these results were insensitive to variations of the other PMM parameters within the ranges from their mean values given in Table 1.
[41] The PMM provides an excellent first-order fit to the MESSENGER observations, with an RMS misfit of less than $20 \mathrm{nT}$ globally. Residual field strengths are typically less than $50 \mathrm{nT}$ compared with maximum field strengths in the observations of up to $500 \mathrm{nT}$. The results show that the 
magnetopause field strength ranges from $10 \%$ to $50 \%$ of the dipole field strength at observation locations on the dayside and at nightside latitudes north of $60^{\circ} \mathrm{N}$.

[42] Residual signatures include time-invariant contributions from unmodeled magnetopause fields resulting from the assumed paraboloid shape for the magnetopause, which has too great a flaring on the nightside. The subsolar distance varies with an 88 day period due to changes in $P_{\text {ram }}$ during Mercury's highly eccentric orbit. Orbit-to-orbit changes in the magnetopause boundary position were also observed. Interorbit and intraorbit differences in the observed magnetospheric fields are most obvious in the tail region, likely related to tail loading and unloading [Slavin et al., 2010] and reconnection. Establishment of an empirically derived activity index for the MAG data will greatly aid subselection of orbits for specific studies, such as quiet orbits for internal field modeling and orbits with substantial high-frequency variability for studies of reconnection. Examination of the residuals from the PMM in the bodyfixed frame suggests persistent structure in the radial field at high northern latitudes. Globally, however, the residual signatures observed to date are dominated by magnetospheric processes, confirming the dynamic nature of Mercury's magnetosphere.

[43] Acknowledgments. The MESSENGER project is supported by the NASA Discovery Program under contracts NAS5-97271 to The Johns Hopkins University Applied Physics Laboratory and NASW-00002 to the Carnegie Institution of Washington. C.L.J. and M.E.P. are supported by MESSENGER Participating Scientist grants NNX11AB84G and NNH08CC05C. R.M.W. and C.L.J. acknowledge support from the Natural Sciences and Engineering Research Council of Canada. I.I.A. is supported by the Russian Foundation for Basic Research grants 11-05-00894-a and 12-02-92600-KO_a and the European FP7 project IMPEx (262863). We thank three anonymous reviewers for helpful comments.

\section{References}

Alexeev, I. I., and V. P. Shabansky (1972), A model of a magnetic field in the geomagnetosphere, Planet. Space Sci., 20, 117-133, doi:10.1016/ 0032-0633(72)90146-8.

Alexeev, I. I., E. S. Belenkaya, S. Y. Bobrovnikov, J. A. Slavin, and M. Sarantos (2008), Paraboloid model of Mercury, J. Geophys. Res., 113, A12210, doi:10.1029/2008JA013368.

Alexeev, I. I., et al. (2010), Mercury's magnetospheric magnetic field after the first two MESSENGER flybys, Icarus, 209, 23-39, doi:10.1016/ j.icarus.2010.01.024.

Anderson, B. J., M. H. Acuña, D. A. Lohr, J. Scheifele, A. Raval, H. Korth, and J. A. Slavin (2007), The Magnetometer instrument on MESSENGER, Space Sci. Rev., 131, 417-450, doi:10.1007/s11214-007-9246-7.

Anderson, B. J., M. H. Acuña, H. Korth, M. E. Purucker, C. L. Johnson, J. A. Slavin, S. C. Solomon, and R. L. McNutt Jr. (2008), The magnetic field of Mercury: New constraints on structure from MESSENGER, Science, 321, 82-85, doi: $10.1126 /$ science. 1159081 .

Anderson, B. J., et al. (2010), The magnetic field of Mercury, Space Sci. Rev., 152, 307-339, doi:10.1007/s11214-009-9544-3.

Anderson, B. J., C. L. Johnson, H. Korth, M. E. Purucker, R. M. Winslow, J. A. Slavin, S. C. Solomon, R. L. McNutt Jr., J. M. Raines, and T. H. Zurbuchen (2011), The global magnetic field of Mercury from MESSENGER orbital observations, Science, 333, 1859-1862, doi:10.1126/ science. 1211001

Anderson, B. J., C. L. Johnson, H. Korth, R. M. Winslow, J. E. Borovsky, M. E. Purucker, J. A. Slavin, S. C. Solomon, M. T. Zuber, and R. L. McNutt Jr. (2012), Low-degree structure in Mercury's planetary magnetic field, J. Geophys. Res., doi:10.1029/2012JE004159, in press.

Belenkaya, E. S., S. Y. Bobrovnikov, I. I. Alexeev, V. V. Kalegaev, and S. W. H. Cowley (2005), A model of Jupiter's magnetospheric magnetic field with variable magnetopause flaring, Planet. Space Sci., 53, 863-872, doi:10.1016/j.pss.2005.03.004.

Benna, M., et al. (2010), Modeling of the magnetosphere of Mercury at the time of the first MESSENGER flyby, Icarus, 209, 3-10, doi:10.1016/ j.icarus.2009.11.036.
Christensen, U. R. (2006), A deep dynamo generating Mercury's magnetic field, Nature, 444, 1056-1058, doi:10.1038/nature05342.

Christensen, U. R., and J. Wicht (2008), Models of magnetic field generation in partly stable planetary cores: Applications to Mercury and Saturn, Icarus, 196, 16-34, doi:10.1016/j.icarus.2008.02.013.

Connerney, J. E. P., and N. F. Ness (1988), Mercury's magnetic field and interior, in Mercury, edited by F. Vilas, C. R. Chapman, and M. S. Matthews, pp. 494-513, Univ. of Ariz. Press, Tucson.

Glassmeier, K.-H., H.-U. Auster, and U. Motschmann (2007a), A feedback dynamo generating Mercury's magnetic field, Geophys. Res. Lett., 34, L22201, doi:10.1029/2007GL031662.

Glassmeier, K.-H., J. Grosser, U. Auster, D. Constantinescu, Y. Narita, and S. Stellmach (2007b), Electromagnetic induction effects and dynamo action in the Hermean system, Space Sci. Rev., 132, 511-527, doi:10.1007/s11214-007-9244-9.

Grosser, J., K.-H. Glassmeier, and A. Stadelmann (2004), Induced magnetic field effects at planet Mercury, Planet. Space Sci., 52, 1251-1260, doi:10.1016/j.pss.2004.08.005.

Heimpel, M., J. M. Aurnou, F. M. Al-Shamali, and N. Gomez Perez (2005), A numerical study of dynamo action as a function of spherical shell geometry, Earth Planet. Sci. Lett., 236, 542-557, doi:10.1016/ j.epsl.2005.04.032.

Heyner, D., J. Wicht, N. Gómez-Pérez, D. Schmitt, H.-U. Auster, and K.-H. Glassmeier (2011), Evidence from numerical experiments for a feedback dynamo generating Mercury's magnetic field, Science, 334, 1690-1693, doi: $10.1126 /$ science. 1207290 .

Kabin, K., T. I. Gombosi, D. L. DeZeeuw, and K. G. Powell (2000), Interaction of Mercury with the solar wind, Icarus, 143, 397-406, doi:10.1006/icar.1999.6252.

Korth, H., B. J. Anderson, M. H. Acuña, J. A. Slavin, N. A. Tsyganenko, S. C. Solomon, and R. L. McNutt Jr. (2004), Determination of the properties of Mercury's magnetic field by the MESSENGER mission, Planet. Space Sci., 52, 733-746, doi:10.1016/j.pss.2003.12.008.

Korth, H., B. J. Anderson, J. M. Raines, J. A. Slavin, T. H. Zurbuchen, C. L. Johnson, M. E. Purucker, R. M. Winslow, S. C. Solomon, and R. L. McNutt Jr. (2011), Plasma pressure in Mercury's equatorial magnetosphere derived from MESSENGER Magnetometer observations, Geophys. Res. Lett., 38 , L22201, doi:10.1029/2011GL049451.

Korth, H., B. J. Anderson, C. L. Johnson, R. M. Winslow, J. A. Slavin, M. E. Purucker, S. C. Solomon, and R. L. McNutt Jr. (2012), Characteristics of the plasma distribution in Mercury's equatorial magnetosphere derived from MESSENGER Magnetometer observations, J. Geophys. Res., doi:10.1029/2012JA018052, in press.

Manglik, A., J. Wicht, and U. R. Chistensen (2010), A dynamo model with double diffusive convection for Mercury's core, Earth Planet. Sci. Lett., 289, 619-628, doi:10.1016/j.eps1.2009.12.007.

Margot, J.-L., S. J. Peale, R. F. Jurgens, M. A. Slade, and I. V. Holin (2007), Large longitude libration of Mercury reveals a molten core, Science, 316 , 710-714, doi:10.1126/science. 1140514

Margot, J.-L., S. J. Peale, S. C. Solomon, S. A. Hauck II, F. D. Ghigo, R. F. Jurgens, M. Yseboodt, J. D. Georgini, S. Padovan, and D. B. Campbell (2012), Mercury's moment of inertia from spin and gravity data, J. Geophys. Res., 117, E00L09, doi:10.1029/2012JE004161.

Moldovan, R., B. J. Anderson, C. L. Johnson, J. A. Slavin, H. Korth, M. E. Purucker, and S. C. Solomon (2011), Mercury's magnetopause and bow shock from MESSENGER observations, abstract EPSC-DPS2011-674 presented at EPSC-DPS Joint Meeting, Eur. Planet. Sci. Congr., Nantes, France.

Ness, N. F., K. W. Behannon, R. P. Lepping, Y. C. Whang, and K. H. Schatten (1974), Magnetic field observations near Mercury: Preliminary results, Science, 185, 151-160, doi:10.1126/science.185.4146.151.

Ness, N. F., K. W. Behannon, R. P. Lepping, and Y. C. Whang (1975), Magnetic field of Mercury confirmed, Nature, 255, 204-205, doi: $10.1038 / 255204 \mathrm{a} 0$.

Odstrcil, D. (2003), Modeling 3-D solar wind structure, Adv. Space Res., 32, 497-506, doi:10.1016/S0273-1177(03)00332-6.

Paschmann, G., and P. W. Daly (Eds.) (1998), Analysis Methods for Multispacecraft Data, ISSI Sci. Rep. Ser., vol. 1, 491 pp., Eur. Space Agency, Noordwijk, Netherlands.

Purucker, M. E., T. J. Sabaka, S. C. Solomon, B. J. Anderson, H. Korth, M. T. Zuber, and G. A. Neumann (2009), Mercury's internal magnetic field: Constraints on large- and small-scale fields of crustal origin, Earth Planet. Sci. Lett., 285, 340-346, doi:10.1016/j.eps1.2008.12.017.

Purucker, M. E., et al. (2012), Evidence for a crustal magnetic signature on Mercury from MESSENGER Magnetometer observations, Lunar Planet. Sci., 42, Abstract 1297.

Shue, J.-H., J. K. Chao, H. C. Fu, C. T. Russell, P. Song, K. K. Khurana, and H. J. Singer (1997), A new functional form to study the solar wind 
control of the magnetopause size and shape, J. Geophys. Res., 102, 94979511, doi:10.1029/97JA00196.

Slavin, J. A., et al. (2010), MESSENGER observations of extreme loading and unloading of Mercury's magnetic tail, Science, 329, 665-668, doi: $10.1126 /$ science. 1188067 .

Slavin, J. A., et al. (2012), MESSENGER observations of a flux-transferevent shower at Mercury, J. Geophys. Res., 117, A00M06, doi:10.1029/ 2012JA017926.

Stanley, S., J. Bloxham, and W. E. Hutchison (2005), Thin shell dynamo models consistent with Mercury's weak observed magnetic field, Earth Planet. Sci. Lett., 234, 27-38, doi:10.1016/j.eps1.2005.02.040.

Takahashi, F., and M. Matsushima (2006), Dipolar and non-dipolar dynamos in thin spherical shell geometry with implications for the magnetic field of Mercury, Geophys. Res. Lett., 33, L10202, doi:10.1029/ 2006GL025792.

Trávníček, P. M., P. Hellinger, and D. Schriver (2007), Structure of Mercury's magnetosphere for different pressure of the solar wind: Threedimensional hybrid simulations, Geophys. Res. Lett., 34, L05104, doi:10.1029/2006GL028518.

Tsyganenko, N. A. (1995), Modeling the Earth's magnetospheric magnetic field confined within a realistic magnetopause, J. Geophys. Res., 100 5599-5612, doi:10.1029/94JA03193.
Tsyganenko, N. A., and M. I. Sitnov (2005), Modeling the dynamics of the inner magnetosphere during strong geomagnetic storms, J. Geophys. Res., 110, A03208, doi:10.1029/2004JA010798.

Uno, H., C. L. Johnson, B. J. Anderson, H. Korth, and S. C. Solomon (2009), Mercury's internal magnetic field: Constraints from regularized inversions, Earth Planet. Sci. Lett., 285, 328-339, doi:10.1016/ j.epsl.2009.02.032.

Vilim, R., S. Stanley, and S. A. Hauck II (2010), Iron snow zones as a mechanism for generating Mercury's weak observed magnetic field, J. Geophys. Res., 115, E11003, doi:10.1029/2009JE003528.

Winslow, R. M., C. L. Johnson, B. J. Anderson, H. Korth, J. A. Slavin, M. E. Purucker, and S. C. Solomon (2012), Observations of Mercury's northern cusp region with MESSENGER's Magnetometer, Geophys. Res. Lett., 39, L08112, doi:10.1029/2012GL051472.

Zurbuchen, T. H., J. M. Raines, G. Gloecker, S. M. Krimigis, J. A. Slavin, P. L. Koehn, R. M. Killen, A. L. Sprague, R. L. McNutt Jr., and S. C. Solomon (2011), MESSENGER observations of the spatial distribution of planetary ions near Mercury, Science, 333, 1862-1865, doi:10.1126/ science. 1211302 